ARTICLE

\title{
Ethylene-independent signaling by the ethylene precursor ACC in Arabidopsis ovular pollen tube attraction
}

\author{
Wangshu Mou (10) 1,2, Yun-Ting Kao (10) 1,4, Erwan Michard,4, Alexander A. Simon (10 1,4, Dongdong Li (i) 1,2,

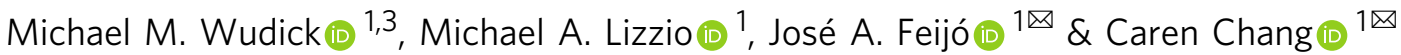

The phytohormone ethylene has numerous effects on plant growth and development. Its immediate precursor, 1-aminocyclopropane-1-carboxylic acid (ACC), is a non-proteinogenic amino acid produced by ACC SYNTHASE (ACS). ACC is often used to induce ethylene responses. Here, we demonstrate that ACC exhibits ethylene-independent signaling in Arabidopsis thaliana reproduction. By analyzing an acs octuple mutant with reduced seed set, we find that ACC signaling in ovular sporophytic tissue is involved in pollen tube attraction, and promotes secretion of the pollen tube chemoattractant LURE1.2. ACC activates $\mathrm{Ca}^{2+}$-containing ion currents via GLUTAMATE RECEPTOR-LIKE (GLR) channels in root protoplasts. In COS-7 cells expressing moss PpGLR1, ACC induces the highest cytosolic $\mathrm{Ca}^{2+}$ elevation compared to all twenty proteinogenic amino acids. In ovules, ACC stimulates transient $\mathrm{Ca}^{2+}$ elevation, and $\mathrm{Ca}^{2+}$ influx in octuple mutant ovules rescues LURE1.2 secretion. These findings uncover a novel ACC function and provide insights for unraveling new physiological implications of ACC in plants.

\footnotetext{
${ }^{1}$ Department of Cell Biology and Molecular Genetics, University of Maryland, College Park, MD, USA. ${ }^{2}$ Present address: Division of Crop Biotechnics, Department of Biosystems, University of Leuven, Leuven, Belgium. ${ }^{3}$ Present address: Institute for Molecular Physiology, Heinrich-Heine-Universität Düsseldorf, Düsseldorf, Germany. ${ }^{4}$ These authors contributed equally: Yun-Ting Kao, Erwan Michard, Alexander A. Simon. ${ }^{凶}$ email: jfeijo@umd.edu; carenc@umd.edu
} 
thylene is a plant hormone that induces numerous physiological responses in the growth and development of flowering plants ${ }^{1}$. Ethylene biosynthesis in flowering plants starts with the synthesis of 1-aminocyclopropane-1-carboxylic acid (ACC) (a non-proteinogenic amino acid) from Sadenosylmethionine via the enzyme ACC SYNTHASE (ACS), followed by conversion of ACC to ethylene by the enzyme ACC OXIDASE (ACO $)^{2}$. Responses to ethylene are often assessed by treating plants with ACC, due to the relative ease of application and rapid conversion of ACC to ethylene ${ }^{2}$. However, exceptions to this rule, such as ethylene-independent ACC responses in roots $^{3-5}$, guard cells ${ }^{6}$, and reproduction ${ }^{7}$ have been reported. In the latter case, an Arabidopsis thaliana octuple mutant of all eight functional ACS genes was generated by expressing an artificial microRNA (amiRNA) that reduced expression of both ACS8 and ACS11 in an acs hextuple knockout mutant background ${ }^{7}$. As expected, the acs octuple mutant exhibited reduced levels of ethylene and displayed phenotypes similar to those of ethyleneinsensitive mutants ${ }^{7}$. However, the octuple mutant also had reduced seed set, which was not observed in the acs hextuple mutant nor alleviated by ethylene treatment ${ }^{7}$, raising the possibility that ACC itself plays a role in reproduction.

In angiosperm reproduction, the success of the progamic phase is critically dependent on the final steps of attraction and targeting of pollen tubes to the ovules, followed by pollen tube rupture to release the sperm cells ${ }^{8}$. While it is generally accepted that the first task implies the secretion of small chemoattractant peptides by the embryo $\mathrm{sac}^{8,9}$, little is known regarding what triggers the secretion and sequential trafficking of the peptides from the synergid cells to the filiform apparatus. This secretion must be well synchronized with pollen tube progression through the pistil, with theoretical predictions suggesting that the redirection of pollen tube growth requires adequate timing of secretion and diffusion of the attractants ${ }^{10,11}$.

Arguments for a role of $\mathrm{Ca}^{2+}$ during plant reproduction have long been proposed ${ }^{12}$ and live imaging has provided circumstantial evidence for a role of $\mathrm{Ca}^{2+}$ signaling during pollen tube/ ovule interaction ${ }^{13-15}$. Critically, our lack of knowledge concerning the nature and regulation of the $\mathrm{Ca}^{2+}$ channels mediating these interactions has hampered an understanding of $\mathrm{Ca}^{2+}$ signaling during reproduction. Glutamate Receptor-Like (GLR) channels, which are implicated in diverse processes involving ion signaling in plants ${ }^{16}$, have been associated with plant reproduction from mosses to angiosperms ${ }^{17-20}$. However, their genetic redundancy and the lack of a comprehensive characterization of critical channel properties, such as ion and ligand selectivity ${ }^{16}$, have made them refractory to mechanistic association with other aspects of reproduction. Of relevance, although GLRs can be stimulated by various amino acids, the exact nature of their regulation in terms of their physiological gating ligands is unclear $^{10}$

Here, we analyze the basis of the acs octuple mutant seed set defect, finding that ACC, but not ethylene, plays a signaling role in the sporophytic tissue of the ovule effecting pollen tube attraction. Moreover, ACC promotes the ovular secretion of a known pollen tube chemoattractant, LURE1.2 $2^{21,22}$, suggesting a possible basis for defective pollen tube attraction. We provide evidence that ACC is capable of gating plant GLR channels, and furthermore, is more potent than any proteinogenic amino acid in inducing a GLR-dependent cytosolic $\mathrm{Ca}^{2+}$ elevation in a heterologous COS-7 cell system. In ovules, ACC treatment triggers a transient $\mathrm{Ca}^{2+}$ elevation, and treatment with a $\mathrm{Ca}^{2+}$ ionophore triggers LURE1.2 secretion. These findings suggest a model in which ACC stimulates GLR-dependent $\mathrm{Ca}^{2+}$ elevation, which in turn promotes LURE1 secretion and pollen tube attraction.
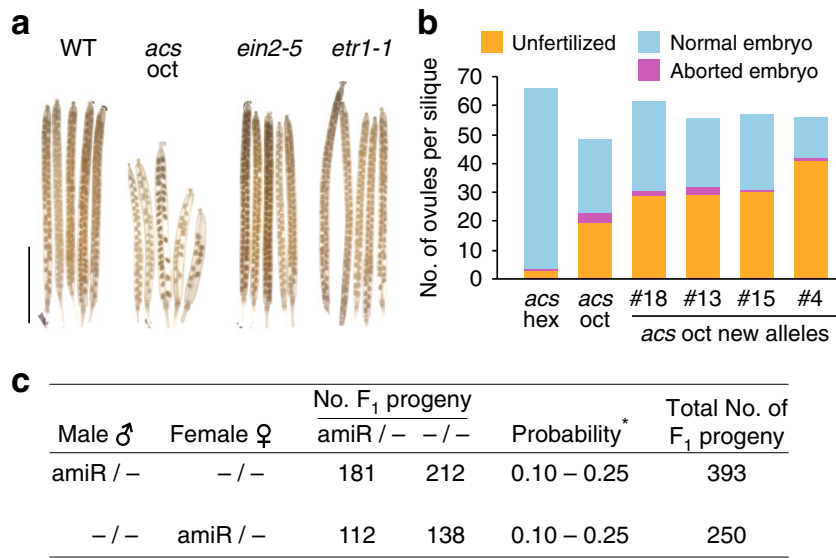

* Probability (chi-square test) of obtaining by chance the observed variation from an expected 1:1 ratio for normal gametophytes d

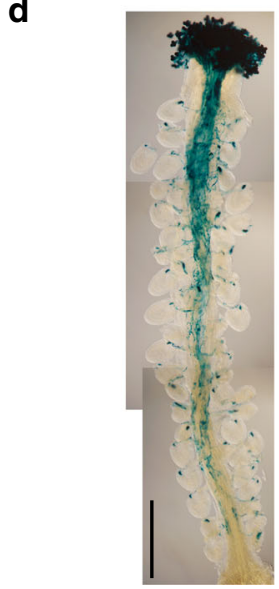

Male of proLat52:GUS

Female $q \quad$ WT

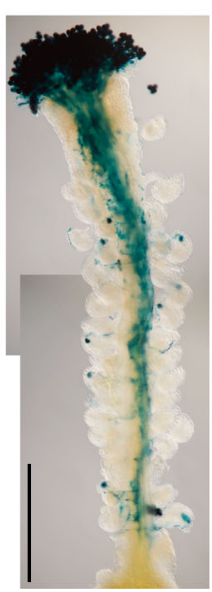

proLat52:GUS

acs oct

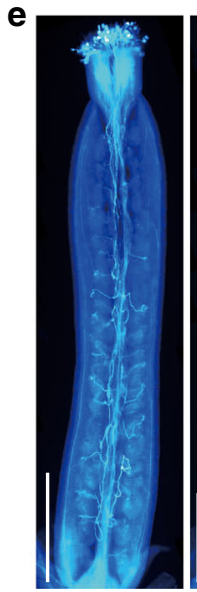

WT

$\stackrel{\times}{\text { WT }}$

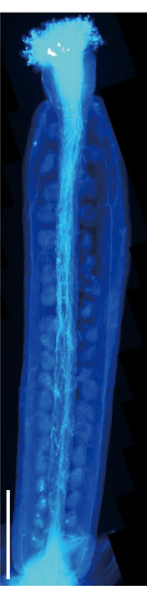

WT

acs oct
Fig. 1 The female sporophyte of the acs octuple mutant is defective in pollen tube targeting. a Representative siliques of the acs octuple (oct) mutant, wild type (WT), and ethylene-insensitive mutants (ein2-5 and etr1-1). Scale bar, $5 \mathrm{~mm}$. b Average number of post-pollination ovule fates per silique in the original acs oct mutant ${ }^{7}$ and four independently generated acs oct mutant alleles compared to their genetic background, the acs hextuple (hex). $n=5$ siliques per genotype, except $n=6$ for Line 18. (For statistical data see Supplementary Fig. 1e-g.) c Reciprocal crosses between WT and the hemizygous amiRNA line. $n=10$ crosses in each direction. d Representative GUS staining of WT pollen tubes expressing LAT52:GUS in dissected hand-pollinated pistils of the WT versus acs oct mutant. A dot of GUS staining within the ovule indicates fertilization by the pollen. Scale bar, $500 \mu \mathrm{m}$. e Representative images of aniline blue-stained WT pollen tubes in hand-pollinated pistils of the WT versus acs oct mutant. Scale bar, $500 \mu \mathrm{m}$.

\section{Results}

Fewer acs octuple mutant ovules are fertilized. We first confirmed that strong ethylene-insensitive mutants have normal seed set in contrast to the acs octuple mutant (Fig. 1a; Supplementary Fig. 1a). We then verified the reduced seed set phenotype of the acs octuple mutant by generating four new octuple mutant alleles, using a different amiRNA sequence to target ACS 8 and ACS11 in the acs hextuple mutant background. The new octuple mutant alleles produced the same reduced ethylene levels as the original octuple mutant (Supplementary Fig. 1b). All of the octuple mutant alleles produced shorter siliques with fewer seeds (Supplementary Fig. 1c, d) and had slightly fewer ovules per pistil compared to the hextuple mutant (Fig. 1b; Supplementary 
Fig. 1c). The ovule number, however, did not account for the reduced seed set. By scoring the post-pollination fates of ovules, we uncovered a high percentage of unfertilized ovules per pistil $(40-73 \%)$ and a low percentage of aborted embryos per pistil (0.7-7.05\%) (Fig. 1b; Supplementary Fig. 1e-g). In contrast to the reported embryonic lethality (aborted embryos) in the acs octuple mutant ${ }^{7}$, these findings indicated that the underlying defect in reduced seed set occurs prior to fertilization.

For further verification of the mutant phenotype, we partially rescued the original acs octuple mutant by co-expressing proACS8:ACS8m and proACS11:ACS11m, each carrying silent point mutations to avoid targeting by the amiRNA. The partially rescued line had wild-type levels of ethylene (Supplementary Fig. 1b), as well as longer siliques (Supplementary Fig. 2a, b) and a higher number of fertilized ovules (Supplementary Fig. 2c-f) than the octuple mutant. In all subsequent experiments we used the original acs octuple mutant ${ }^{7}$.

The acs mutant female sporophyte confers reduced seed set. Reciprocal crosses between the acs octuple mutant and wild type indicated that the female is responsible for the reduced seed set (Supplementary Fig. 3a-d). However, the floral organs of the acs octuple mutant showed no obvious morphological defects (Supplementary Fig. 3e). Hand pollination of the octuple mutant 24, 36 , and $48 \mathrm{~h}$ after emasculating flowers at floral stage $12 \mathrm{~b}^{23}$ did not rescue the seed number, suggesting the mutant female is not developmentally delayed (Supplementary Fig. $4 a-d$ ). To evaluate whether the defect lies with the female gametophyte or sporophyte, we carried out reciprocal crosses using acs hextuple mutant plants that were hemizygous for the original amiRNA. When we hand-pollinated wild-type pistils using pollen from the hemizygous plants, and vice versa, the amiRNA transgene (representing male and female acs octuple gametophytes, respectively) segregated $1: 1$ in the $F_{1}$, indicating that neither the male or female gametophyte is responsible for the seed set defect (Fig. 1c). However, the total number of $F_{1}$ progeny was significantly lower when wild-type pollen was used to hand-pollinate hemizygous pistils $\left(250 \mathrm{~F}_{1}\right.$ compared to $393 \mathrm{~F}_{1}$ for the reciprocal cross, $P<0.01$ ) (Fig. 1c). These results strongly suggested that the female sporophyte is responsible for the pre-fertilization defect in the acs octuple mutant and indicated that the hemizygous amiRNA in the mutant is dominant, as expected. Consistent with a dominant sporophytic defect, the amiRNA segregated $3: 1(P=$ $0.25-0.50)$ in the self-progeny of the hemizygote.

acs mutant pistils reduce pollen tube targeting of ovules. One of the most important events required for successful fertilization is the near-orthogonal change in growth trajectory of pollen tubes when exiting the transmitting tract to grow towards the ovule . $^{\text {. }}$ This altered trajectory is thought to be triggered by chemotropic factors that emanate from the embryo $\mathrm{sac}^{8,9}$. When we handpollinated the wild type versus acs octuple mutant using wild-type pollen expressing a pollen-specific $\beta$-glucuronidase (GUS) reporter, we observed in planta pollen tube targeting of $93.8 \% \pm$ $3.2 \%$ of wild-type ovules ( $n=12$ pistils) compared to only $45.9 \%$ $\pm 15.7 \%$ of octuple mutant ovules ( $n=19$ pistils). This reduced level of targeting (Fig. 1d) is consistent with the proportion of seeds observed in the mutant. Likewise, fewer wild-type pollen tubes appeared to turn towards the ovules in octuple mutant pistils than in wild-type pistils (Fig. 1e). Pollen tubes of strong ethylene-insensitive mutants behaved similarly, turning poorly towards octuple mutant ovules (Supplementary Fig. 5a, b), thus ruling out ethylene as a chemoattractant. Conversely, an ethyleneinsensitive female still attracted pollen tubes (Supplementary Fig. 5c), consistent with ethylene signaling in the female organs not playing a role in the mutant phenotype. These results suggested that the acs octuple mutant pistil is defective in pollen tube guidance.

acs mutant ovules are defective in attracting pollen tubes. To identify which parts of the mutant pistil affect pollen tube targeting, we turned to the semi-in vivo pollen tube guidance assay $^{24}$. The assay involves placing an excised stigma-style of a hand-pollinated pistil horizontally on agar medium, allowing the pollen tubes to grow out of the cut style and along the agar surface towards excised ovules that are prearranged on the agar surface (Fig. 2a). Using this assay, we challenged wild-type pollen tubes emerging from the cut style with both wild-type and acs octuple mutant ovules placed on opposite sides of a midline extending from the cut style (Supplementary Fig. 6). Pollen tubes were significantly less attracted to the mutant ovules (Fig. 2b); for example, in 19 assays, a total of 227 pollen tubes (56\%) turned towards the wild-type ovules, whereas 140 pollen tubes $(35 \%)$ turned towards the mutant ovules $(P<0.01)$ (Table 1$)$. There were no detectable effects on pollen tube turning when using pistils and/or pollen from the acs octuple mutant (Table 1). These findings indicated that the ovules of the acs octuple mutant are defective in pollen tube attraction.

Pollen tube attraction is rescued by ACC and not by ethylene. Notably, we rescued pollen tube attraction by pretreating acs octuple mutant ovules with ACC $(1 \mu \mathrm{M})$ just prior to placing them in the semi-in vivo assay (Fig. $2 \mathrm{c}$, Table 1 ). In contrast, pretreating the ovules with ethylene $(10 \mathrm{ppm})$ did not rescue the defect (Fig. 2d, Table 1), nor did conducting the assay in the presence of ethylene $(10 \mathrm{ppm})$ (Table 1$)$. Pretreating wild-type ovules with ACC $(1 \mu \mathrm{M})$ did not enhance their ability to attract pollen tubes (Fig. 2e, Table 1), and pollen tubes showed no preference for agar beads pre-soaked in ACC $(1 \mu \mathrm{M})$ (Fig. 2f, Table 1), indicating a requirement for the ovule and excluding the possibility that ACC itself is a chemoattractant. From these results, we concluded that ACC, not ethylene, is an ovule-derived signal involved in mediating pollen tube attraction in Arabidopsis.

acs octuple mutant ovules are defective in LURE1.2 secretion. Cysteine-rich LURE1 peptides are pollen tube chemoattractants sufficient to attract pollen tubes in vitro ${ }^{21,22}$. LURE1s are expressed in the synergid cells of the gametophyte, and prior to fertilization, they are trafficked to the filiform apparatus of the synergid cells and secreted by unknown mechanisms to the micropyle and funicular surface of the ovule $\mathrm{e}^{21,22}$. In the acs octuple mutant, floral buds have normal transcript levels of LURE1.1-1.5 (Supplementary Fig. 7a). To examine LURE1 peptide secretion, we stably expressed proLURE1.2:LURE1.2-eGFP in the acs octuple mutant. LURE1.2 has strong chemoattractant activity and is the most abundantly expressed LURE ${ }^{22}$. Shown are three distinct localizations of LURE1.2-eGFP in the acs octuple mutant (Fig. 2g, Supplementary Fig. 7b) and in the wild type (Supplementary Fig. 7b). Interestingly, the LURE1.2-eGFP signal was more frequently observed in the synergid cells of acs octuple mutant ovules (imaged $24-28 \mathrm{~h}$ after emasculating the pistils at floral stage $12 c^{23}$ ) compared to the wild type, indicating a defect in LURE1.2-eGFP trafficking or secretion to the filiform apparatus (Fig. 2h, Supplementary Fig. 7b, c). The relatively low proportion of secreted LURE1.2-eGFP, even in the wild type, could be due to the eGFP fusion. We rescued LURE1.2-eGFP secretion by pretreating octuple mutant ovules with ACC $(1 \mu \mathrm{M})$ (Fig. 2i, Supplementary Fig. 7d), whereas we observed no difference when the ovules were pretreated with ethylene $(10 \mathrm{ppm})$ (Fig. 2j, Supplementary Fig. 7e). Moreover, in an acs octuple 


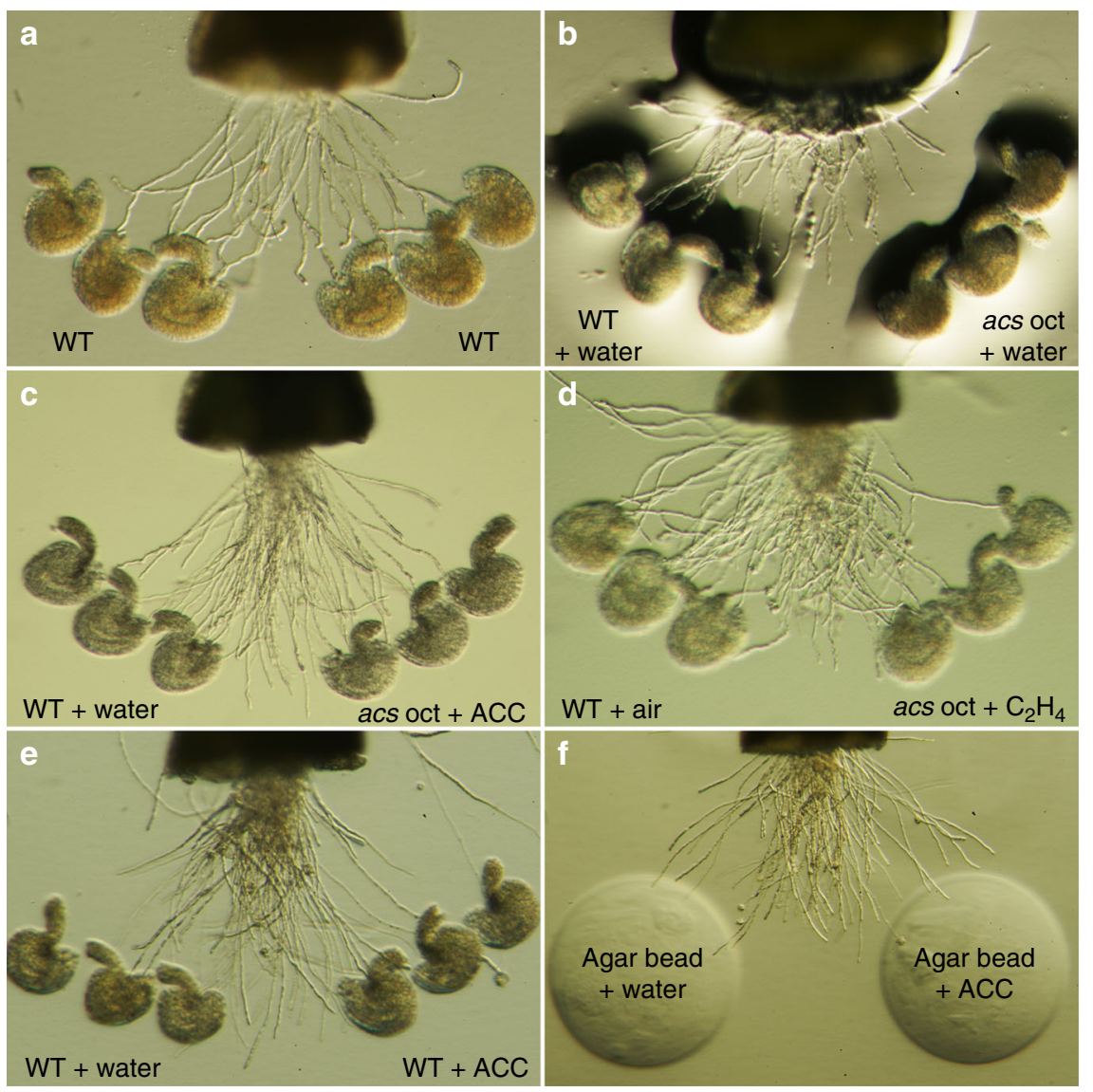

g
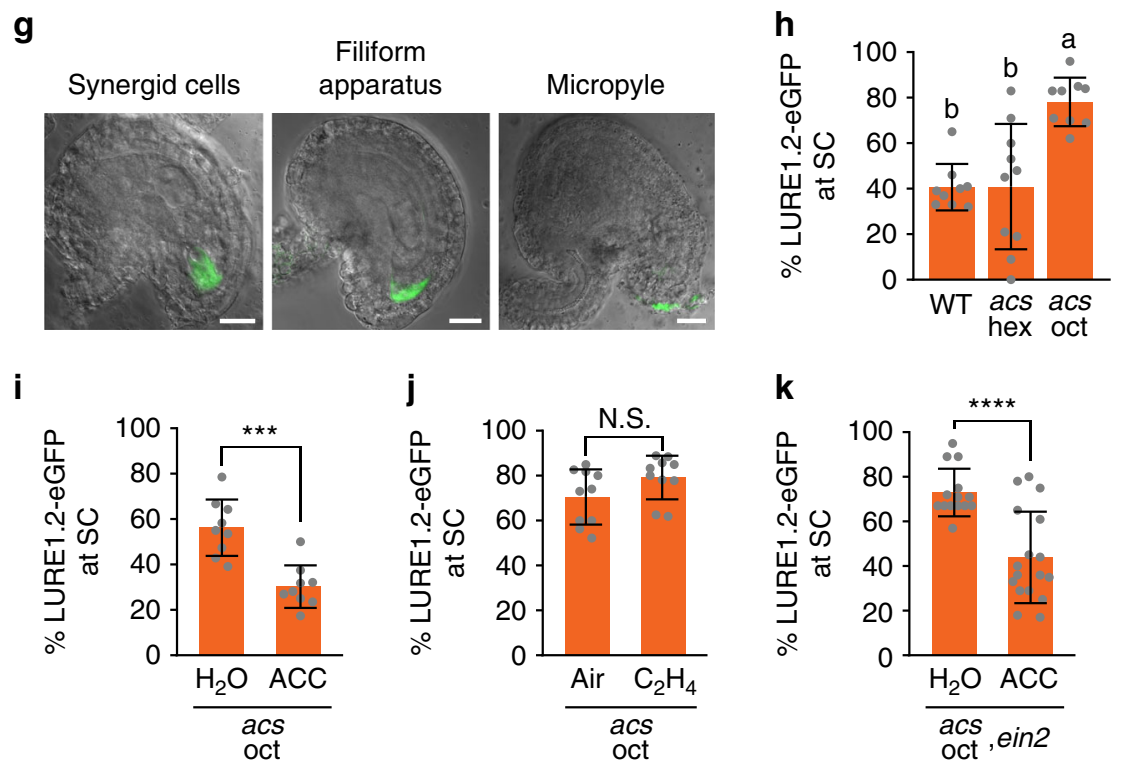

Fig. 2 ACC has an ethylene-independent role in pollen tube attraction and LURE1.2-eGFP localization. a-f Representative images of semi-in vivo pollen tube guidance assays. Pretreatments (+water, $+\mathrm{ACC},+\mathrm{C}_{2} \mathrm{H}_{4}$ ) of ovules and agar beads are indicated. $\mathbf{g}$ Representative images of LURE1.2-eGFP localization in acs oct ovules $24 \mathrm{~h}$ after emasculation of stage 12c floral buds. Scale bar, $20 \mu \mathrm{m}$. h-k Average percentages of synergid cell (SC)-localized LURE1.2-eGFP in ovules per pistil (for ovules in which localization could be determined). Error bars show \pm s.d. $\mathbf{h}$ No treatment; $n=9,10,9$ pistils for WT, acs hex, acs oct, respectively. Different letters indicate significant difference (Welch ANOVA tests with Tamhane's T2 multiple comparisons test $P<0.05$, $\mathrm{df}=2.0, W=29.690)$. i Pretreatment with ACC $(1 \mu \mathrm{M}) ; n=9$ pistils per sample (two-tailed $t$-test, $\left.P=0.0001\left({ }^{\star \star \star}\right), t=4.998, \mathrm{df}=16\right)$. $\mathbf{j}$ Pretreatment with $\mathrm{C}_{2} \mathrm{H}_{4}(10 \mathrm{ppm}) ; n=10$ pistils per sample (two-tailed $t$-test, $P=0.0971$ (N.S.), $\left.t=1.750, \mathrm{df}=18\right)$. k Pretreatment with $\mathrm{ACC}(1 \mu \mathrm{M}) ; n=14$ pistils for $\mathrm{H}_{2} \mathrm{O}, n=17$ pistils for ACC (two-tailed $t$-test with Welch's correction $P<0.0001\left(^{\star \star \star \star}\right), t=5.072, \mathrm{df}=24.96$ ). 
Table 1 Quantification of pollen tube attraction in the semi-in vivo pollen tube guidance assay.

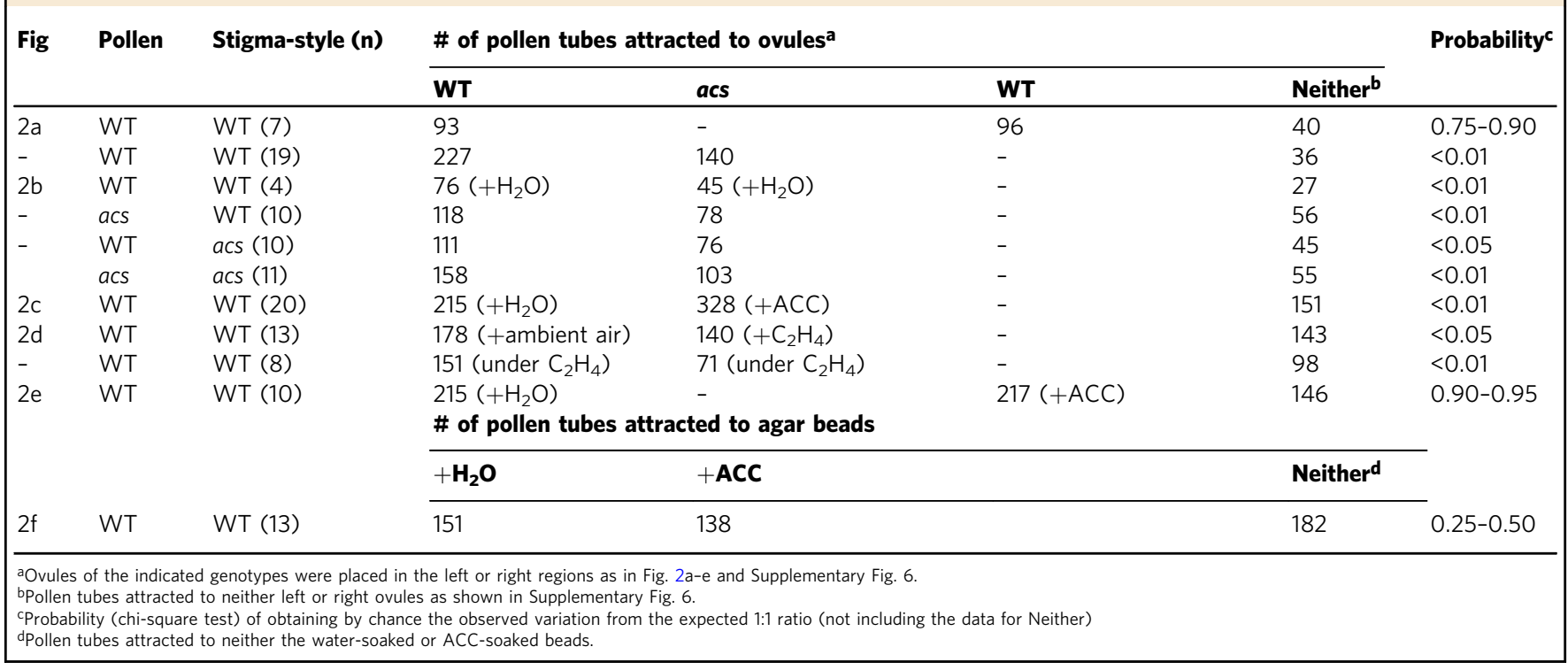

mutant that was rendered ethylene-insensitive by a CRISPR/ Cas9-induced deletion in the EIN2 gene, LURE1.2-eGFP secretion was still rescued by ACC $(1 \mu \mathrm{M})$, ruling out a role for ethylene (Fig. 2k, Supplementary Fig. 7f).

Consistent with ACC being needed in the ovule sporophytic tissue, a GUS reporter fused to the ACS8 promoter was localized to the sporophytic tissue of the ovule (Supplementary Fig. $7 \mathrm{~g}$ ). (Expression of ACS11, the other gene knocked down in the octuple mutant, has not been detected in ovules ${ }^{25}$.) These results suggested that ACC is an inducing factor in LURE1.2 trafficking, and indicate that prevention of LURE1.2 trafficking may contribute to reduced pollen tube guidance toward the micropyle of acs octuple mutant ovules. ACC signaling might affect other pollen tube guidance signals ${ }^{9}$, including proposed long-range ovular signals that have yet to be identified ${ }^{26}$. Given that acs octuple mutants are not null mutants, a more complete loss of ACC is likely to confer additional and/or more severe phenotypes. Nevertheless, ACC in the ovule sporophytic tissue plays a unique signaling role in pollen tube guidance. This role is distinct from the function of ethylene in postfertilization synergid cell death and the pollen tube block in Arabidopsis ${ }^{27}$, and is unlike the role of ethylene in megasporogenesis in tobacco ${ }^{28}$.

ACC is capable of gating plant GLR channels. Although not synthesized in animal tissues, ACC is a partial agonist of ionotropic glutamate receptors (iGluRs), which are ligand-gated ion channels involved in electric and $\mathrm{Ca}^{2+}$ signaling in neurons ${ }^{29-31}$. Given ACC's signaling function in plants, we investigated whether ACC is capable of stimulating the plant homologs of iGluRs known as GLR channels. We tested whether ACC could activate GLR-dependent ion conductance using whole-cell patch-clamp on Arabidopsis root epidermal protoplasts, starting with the highest order $g l r$ mutant available ( $g l r 3.1, g l r 3.2, g l r 3.3, g l r 3.6)$. When treated with ACC, wild-type protoplasts displayed an increase in current amplitude, whereas those of the quadruple $g l r$ mutant (which displayed significantly less current without any ligand than did wild-type protoplasts: Supplementary Fig. 8a, b) were unresponsive to ACC (Fig. 3a, b). Besides this response to ACC, we confirmed these currents using the cation channel inhibitor $\mathrm{Gd}^{3+}(500 \mu \mathrm{M})$, which inhibited $>80 \%$ of the macroscopic current, showing that the ohmic-like current recorded was not an artifactual leak but was rather induced by non-selective channels (Supplementary Fig. 8c). A glr3.1, glr3.2 double mutant still responded to ACC, but the glr3.3 and glr3.6 single and double mutants did not respond (Fig. 3b), suggesting that the ACCinduced current is dependent on AtGLR3.3 and AtGLR3.6. The lack of ACC response in glr3.3 and glr3.6 may be through heteromer formation, as it is well established that iGluR channels in animals are tetramers formed by homo- or heteromerization, with the specific combination of monomers having a determining role with respect to the ligand ${ }^{16,32}$. These results provide proofof-principle that ACC can elicit GLR-mediated, $\mathrm{Ca}^{2+}$-containing currents in a physiological (root protoplast) system.

ACC is the strongest agonist of PpGLR1-driven $\mathrm{Ca}^{2+}$ elevation. Functional GLR ligands in planta remain unclear, although various candidate amino acids have been proposed $16,33,34$. The apparent promiscuity of GLR agonists arguably suggests that GLRs are adapted to functions where they process information associated with various amino acids. This view recently received support from a structural analysis of the AtGLR3.3 ligandbinding domain ${ }^{35}$, raising the possibility that a preferred ligand in plants remains unaccounted for. Because Arabidopsis evolved twenty GLRs (more than humans), angiosperm GLRs constitute a challenging model for elucidating ligand specificity ${ }^{17,33,34}$. In contrast to Arabidopsis, the moss Physcomitrella patens expresses only one GLR ( $P$ G GLR1) in its vegetative tissues ${ }^{18}$, suggesting that $P p$ GLR1 is sufficient to form functional tetra-homomeric channels. Previous characterization of PpGLR1 currents by patchclamp during heterologous COS-7 mammalian cell expression has shown them to be non-selective like those of Arabidopsis GLRs, with important conductance for $\mathrm{Na}^{+}$and a smaller proportion of $\mathrm{Ca}^{2+}$ driven-currents ${ }^{18}$. Of relevance, the magnitude of these $\mathrm{Ca}^{2+}$ currents driven by PpGLR1 were deemed as sufficient to sustain cytosolic $\mathrm{Ca}^{2+}$ elevations with enough magnitude to generate $\mathrm{Ca}^{2+}$-signaling events ${ }^{18}$.

To test ligand potency on $\mathrm{Ca}^{2+}$ elicitation, we thus turned to these same conditions ${ }^{18}$ in COS-7 cells co-expressing PpGLR1 and the $\mathrm{Ca}^{2+}$ sensor Yellow Cameleon $3.6(\mathrm{YC} 3.6)^{36}$, using $\mathrm{Ca}^{2+}$ imaging as a read-out. In these conditions, PpGLR1 induces a cytosolic $\mathrm{Ca}^{2+}$ elevation when cells are exposed to extracellular $\mathrm{Ca}^{2+}$ in the absence of a candidate ligand, but we found that ACC 
a

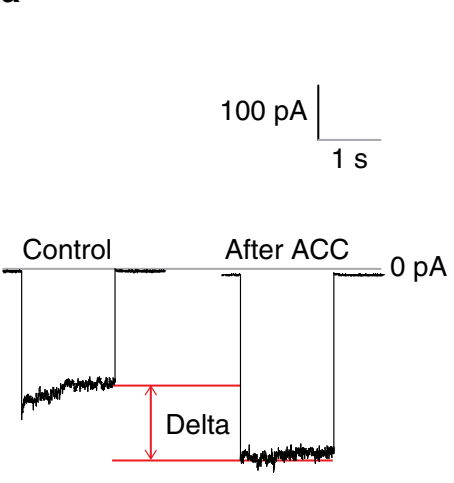

b

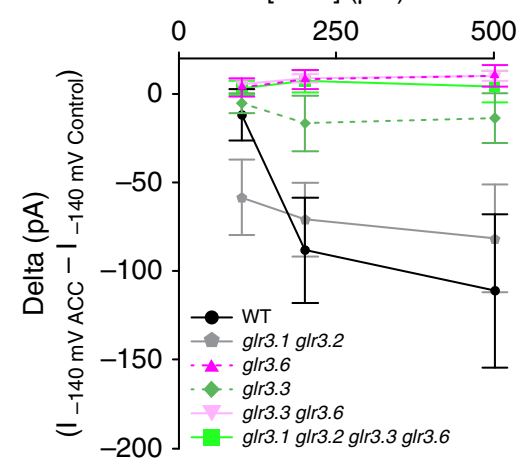

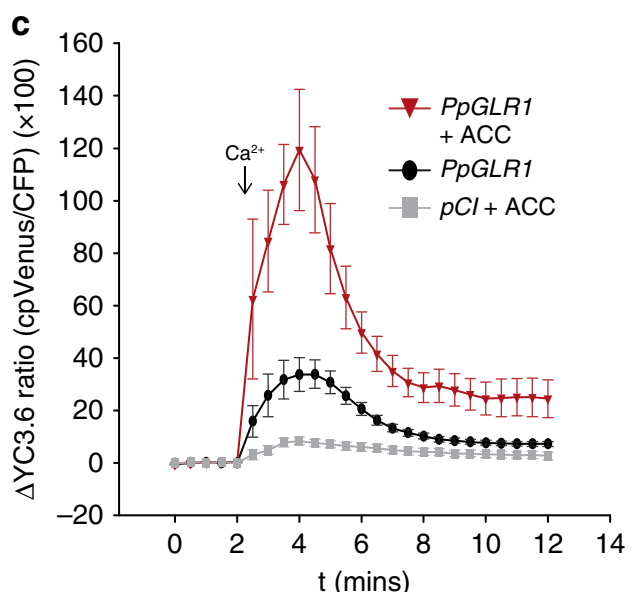

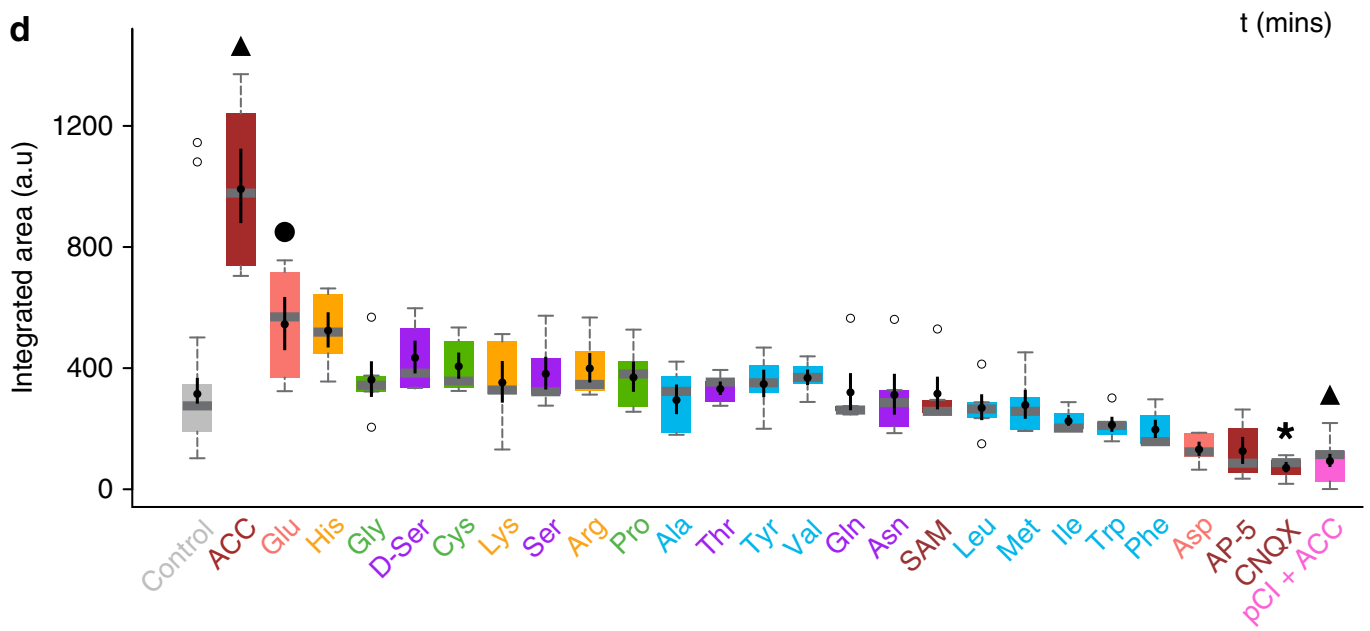

Fig. 3 ACC stimulates GLR-dependent ion transport in Arabidopsis root protoplasts and mammalian COS-7 cells. a Macroscopic currents recorded at $-140 \mathrm{mV}$ under the whole-cell configuration in a WT Arabidopsis root protoplast show an increase (delta) after adding ACC (500 $\mu \mathrm{M}$ ) to the bath solution. b Average currents (delta) induced by ACC $(100,200$, or $500 \mu \mathrm{M})$ in experiments similar to that in a for root protoplasts of WT and glr knockout mutants. WT and glr3.1, glr3.2: $n=5$ for 100, $200 \mu \mathrm{M} \mathrm{ACC,} n=4$ for $500 \mu \mathrm{M} \mathrm{ACC.} \mathrm{Quadruple} \mathrm{mutant:} n=4$ for 100, $200 \mu \mathrm{M} \mathrm{ACC,} n=3$ for $500 \mu \mathrm{M} \mathrm{ACC.} \mathrm{glr3.3,}$ glr3.6: $n=4$ for $100 \mu \mathrm{M} \mathrm{ACC,} n=5$ for 200 and $500 \mu \mathrm{M}$ ACC. glr3.3: $n=4$ for all ACC concentrations. glr3.6: $n=4$ for $100 \mu \mathrm{M}$ ACC, $n=6$ for 200 and $500 \mu \mathrm{M}$ ACC. Error bars show \pm s.e. c Time course of mean cytosolic $\mathrm{Ca}^{2+}$ changes in COS-7 cells expressing PpGLR1 monitored with YC3.6 when applying $\mathrm{Ca}^{2}+(14.5 \mathrm{mM}$; arrow) to the bath solution in the presence of $\mathrm{ACC}(500 \mu \mathrm{M}$; red triangles, $n=6)$ and absence of $A C C$ (black circles, $n=31)$. Cells transfected with empty vector $(\mathrm{pCl})$ were also treated with $\mathrm{ACC}(500 \mu \mathrm{M}$; gray squares, $n=10)$. Statistical significance was determined between the mean of each curve by two-way ANOVA (PpGLR1 + ACC vs. PpGLR1: $P<0.0001 ; P p G L R 1+A C C$ vs. $p C I+A C C: P<0.0001 ; P p G L R 1$ vs. pCI + ACC: $P<$ $0.0001 . F=50.74, d f=2$ ). Error bars show \pm s.e. $\mathbf{d}$ Comparison of $A C C$ to the 20 proteinogenic amino acids based on $\mathrm{Ca}^{2+}$ imaging in COS-7 cells expressing PpGLR1 and YC3.6 as in c. Box plots show changes in cytosolic $\mathrm{Ca}^{2+}$ evaluated by the integrated area under the YC3.6 response curve for each amino acid (top of box is 75th percentile; bottom of box is 25th percentile; horizontal gray bar is median; gray whiskers show the maximum and minimum value within $1.5 \times$ the interquartile range) with outliers (open circles) and mean (solid black circles) \pm s.e. Included are two candidate ligands, D-Serine (DSer) and the ACC precursor S-adenosyl-L-methionine (SAM), and two classic iGluR antagonists, 2-amino-5-phosphonovalerate (AP-5) and 6-cyano-7nitroquinoxaline-2,3-dione disodium salt hydrate (CNQX). $n=31$ for pCI-PpGLR1 control (no amino acid added), $n=6$ for $p C l-P p G L R 1+A C C, n=10$ for $\mathrm{pCl}$ (empty vector) $+\mathrm{ACC}, n=5$ for all other treatments. Statistical significance was determined using Dunnett's test (solid black circle $P<0.1,{ }^{\star} P<0.05$, solid black triangles $P<0.001$ ).

strongly potentiated this response (Fig. 3c). By comparing the responses induced by ACC and all 20 proteinogenic amino acids, ACC was revealed to be the most potent activator of PpGLR1dependent cytosolic $\mathrm{Ca}^{2+}$ elevation (Fig. 3d, Supplementary Fig. 8d). ACC is thus a candidate ligand of the GLR channel family. The range of effects, from strongest activation (ACC) to strongest inhibition (CNQX), is also suggestive of a direct action of the amino acid on PpGLR1, consistent with what is now known from the interaction of amino acids with the ligandbinding domain of AtGLR3.3 $3^{35}$.

ACC induces GLR-mediated $\mathbf{C a}^{2+}$ elevation in ovules. To test whether ACC induces $\mathrm{Ca}^{2+}$ elevations in ovules, we imaged the cytosolic $\mathrm{Ca}^{2+}$ reporter GCaMP3 $3^{37}$ expressed in the ein2-5 mutant. We found that ACC induced a single, strong transient increase in $\mathrm{Ca}^{2+}$ levels in the ovule. This $\mathrm{Ca}^{2+}$ elevation forms in a sporophytic region of the ovule near the termination of the vascular bundles, then spreads to the rest of the ovule. Importantly, this increase was repressed when pretreating ovules with the iGluR antagonist CNQX (Fig. 4a-c, Supplementary Movies 1, 2). CNQX was previously shown to inhibit GLRs without discernible effect on other channels currents ${ }^{16-18}$, which we have now confirmed in PpGLR1 (Fig. 3d). We then pretreated ovules with the $\mathrm{Ca}^{2+}$ ionophore A23187 $(10 \mu \mathrm{M})$, which has been widely used to raise internal $\mathrm{Ca}^{2+}$ levels ${ }^{38}$ and has been convincingly shown to work in plant cells ${ }^{39-41}$. Notably, this treatment was sufficient to rescue the retention of LURE1.2-eGFP in the synergid cells of the acs octuple mutant ovules; we observed less 

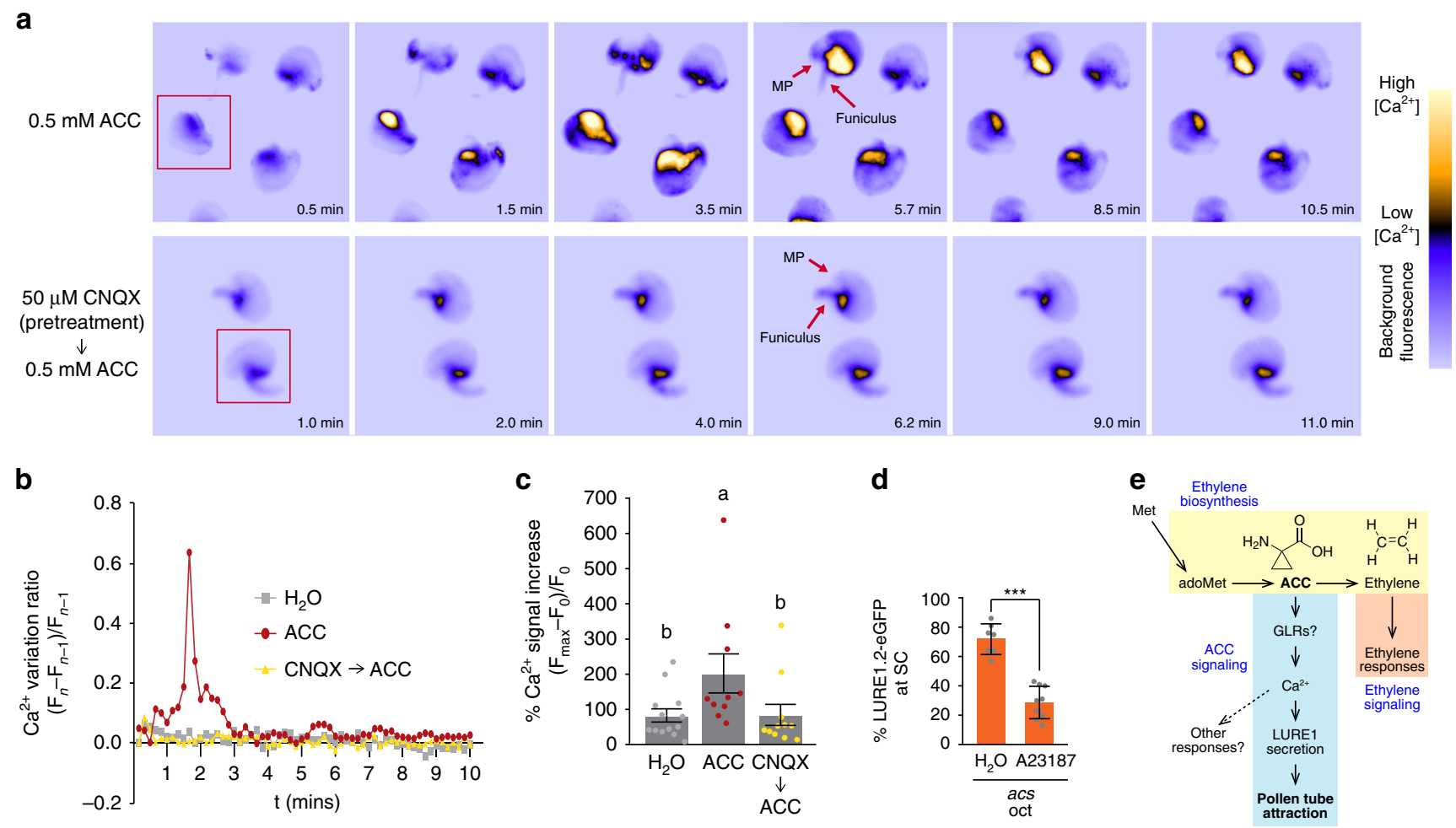

Fig. 4 ACC stimulates CNQX-dependent $\mathbf{C a}^{2+}$ elevation in ovules. a Representative time sequence after applying ACC ( $\left.500 \mu \mathrm{M}\right)$ to ovules expressing cytosolic GCaMP3. Scale reflects arbitrary units of fluorescence (see "Methods"). Top row: time sequence of transient cytosolic Ca ${ }^{2+}$ elevation after adding ACC. The $\mathrm{Ca}^{2+}$ signal first appears near the funiculus (seen from background fluorescence) and expands to the rest of the ovule. Bottom row: similar time sequence after adding ACC $(500 \mu \mathrm{M})$ immediately after preincubation (10 min) with CNQX (50 $\mu$ M). (See Supplementary Movies 1,2 for unprocessed movies.) Red boxes indicate the ovules whose signals are plotted in $\mathbf{b}$. $\mathbf{b} \mathrm{Ca}^{2+}$ variation ratios for the time sequences of ovules in $\mathbf{a}$ (red boxes) and for $\mathrm{ddH}_{2} \mathrm{O}$ as a control. Treatments consist of $\mathrm{ddH}_{2} \mathrm{O}$ (gray square), ACC (red circle), and CNQX to ACC (yellow triangle). c Percentage of $\mathrm{Ca}^{2+}$ signal increase for ovules treated with $500 \mu \mathrm{M} \mathrm{ACC}(n=10), 500 \mu \mathrm{M} \mathrm{ACC}$ (pretreated with $\left.50 \mu \mathrm{M} \mathrm{CNQX} ; n=11\right)$ and ddH $\mathrm{O}_{2}(n=13)$. These data were analyzed with Kruskal-Wallis with Dunn's post hoc test, $\mathrm{df}=2$, Kruskal-Wallis statistic $=9.062$, Error bars show \pm s.e. $\mathbf{d}$ Treatment with the calcium ionophore A23187 rescues LURE1.2-eGFP localization in acs oct ovules. Average percentage of SC-localized LURE1.2-eGFP in acs oct ovules per pistil (for

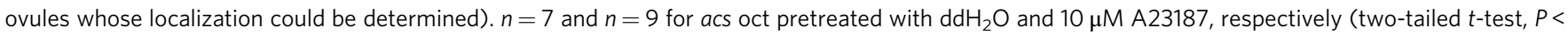
$\left.\left.0.0001{ }^{(* \star \star}\right), t=7.926, \mathrm{df}=14\right)$. Error bars show \pm s.d. e Model of ACC signaling. Besides being the ethylene precursor, $\mathrm{ACC}$ induces $\mathrm{Ca}{ }^{2+}$-dependent LURE1 trafficking and pollen tube attraction by the ovule through an ethylene-independent pathway that might involve GLRs.

LURE1.2-eGFP in the synergid cells and more in the filiform apparatus (Fig. 4d, Supplementary Fig. 9). These findings raise the possibility of a causal link between ACC-induced $\mathrm{Ca}^{2+}$ elevation and pollen tube attraction, in which ACC-induced $\mathrm{Ca}^{2+}$ elevation plays a role in LURE1 trafficking and pollen tube attraction leading to fertilization (Fig. 4e). Given our demonstration that ACC is the most potent elicitor of GLR-mediated $\mathrm{Ca}^{2+}$ elevations, the hypothesis that these channels work as targets of ACC constitutes the most parsimonious interpretation of our data. All four of the AtGLRs tested (Fig. $3 \mathrm{~b}$ ) are highly expressed in ovules ${ }^{42}$.

\section{Discussion}

These findings support the emerging evidence of a signaling function for ACC distinct from its role as the ethylene precursor $^{3-7}$. Seed plants typically contain micromolar levels of ACC, allowing for ACC to simultaneously serve both of these functions. Whether ACC is converted to ethylene depends on the expression and activity of ACO, and can be affected by ACC conjugation to malonyl, $\gamma$-glutamyl, or jasmonyl ${ }^{43}$. We have not ruled out the possibility of signaling by conjugated forms of ACC, the functions of which are unknown. Data suggest non-seed plants do not use ACC as an ethylene precursor ${ }^{44}$, consistent with the absence of ACO homologs in such plants ${ }^{45}$. Yet biosynthesis of ACC appears to have been conserved for several hundred million years prior to the emergence of seed plants ${ }^{45}$. Thus, ACC could have had ancient signaling roles in the plant lineage beyond angiosperms. Interestingly, ACC-induced GLR signaling fits with previous implications that GLRs were conserved in the evolution of land plant-associated cell-cell communication, which is required in fertilization ${ }^{17,18}$. While the triggering of postpollination steps in ovule development has long been described $^{46}$, we now propose a mechanism for the release of chemotropic peptides from the gametophyte controlled by the sporophytic tissue. How the stigma signal reaches the ovules is unknown, but the elicitation of electric potentials along the style following compatible pollination have been reported $47-49$, and such long-range electric communication between organs has been recently associated with GLR function ${ }^{50,51}$. Interestingly, the propagation of these electric potentials occur along the phloem tissue, and our data suggest that ACC-induced $\mathrm{Ca}^{2+}$ signals in the ovule lie at the termination of the vascular bundles in the phloem.

\section{Methods}

Plant materials and general growth conditions. Arabidopsis thaliana ecotype Columbia (Col-0) was the wild type. The acs hextuple (acs2-1, acs4-1, acs5-2, acs61, acs 7-1, acs9-1) and octuple mutants were obtained from the Arabidopsis Biological Resource Center (ABRC) and confirmed by genotyping; acs2-1, acs4-1, acs52 , acs6-1, acs7-1, and acs9-1 alleles were genotyped as described ${ }^{7}$ using T-DNA left border primer "TL-3" (5' GTACATTAAAAACGTCCGCAATGTG-3') (except for acs7-1), and the amiRNA transgene, $(a m i R)$ was genotyped using primers shown in Supplementary Table S1. Except where specified, all analyses of the octuple mutant 
were carried out using the previously published homozygous acs octuple mutant ${ }^{7}$. A transgenic wild-type line expressing the $\beta$-glucuronidase (GUS) reporter from the pollen-specific LAT52 promoter (proLAT52:GUS) ${ }^{52}$ was a gift from Ravishankar Palanivelu (University of Arizona, Tuscon). Double and quadruple $g l r$ mutants were from Edward Farmer (University of Lausanne, Switzerland). The eto1, eol1, eol2 mutant was from Dr. Bram Van de Poel (KU Leuven, Belgium).

To grow plants, surface-sterilized seeds were cold stratified for 3 days on $0.8 \%$ agar medium containing 1x Murashige and Skoog salts (Caisson Labs), then germinated under 16- or 24 -h light $\left(\sim 120 \mathrm{~mol} \mathrm{~m}^{-2} \mathrm{~s}^{-1}\right)$ at $22^{\circ} \mathrm{C}$ except where noted. Ten-day-old seedlings were transferred to soil (2 parts Fafard Fine Germination Mix:1 part Vermiculite) and grown in chambers under 16-h light/8-h dark at $22^{\circ} \mathrm{C}$. Relative humidity was maintained at $45-70 \%$.

Seed number and silique length measurements. Plants of each genotype were grown side-by-side under the same conditions. Siliques at the mature green stage (7-10 days post-pollination) were collected from the middle of the main stem, excluding the first $4-5$ siliques and the last several siliques. To count seeds, the carpel walls were cleared by incubating the siliques in $95 \% \mathrm{EtOH}$ for about 1 week, then photographed under a stereoscopic zoom microscope (Nikon SMZ1000). The number of seeds per silique was counted based on the images, and silique length was determined using Image ${ }^{53}$.

New acs octuple mutant alleles. Plasmid pRS300 ${ }^{54}$ was used as a PCR template to create a new artificial microRNA $(a m i R)$ distinct from that used in Tsuchisaka et al. ${ }^{7}$. We PCR-amplified three DNA fragments, using primers A + IV, III + II and I + B shown in Supplementary Table S1, as described ${ }^{7}$, and cloned the fragments by Gibson assembly into SpeI/KpnI-digested binary vector pMDC $32^{55}$. The construct was transformed into the acs hextuple mutant by the floral dip method ${ }^{56}$ using Agrobacterium tumefaciens strain GV3101 (pMP90) ${ }^{57} \cdot \mathrm{T}_{1}$ transformants were selected on hygromycin B $(15 \mu \mathrm{g} / \mathrm{mL})$ as described ${ }^{58}$ and confirmed by PCRgenotyping of both the hygromycin resistance gene and the amiR sequence, using primers shown in Supplementary Table S1. The $\mathrm{T}_{1}$ plants were used for determining ovule fates. Homozygous $\mathrm{T}_{3}$ progeny were used for ethylene measurements.

Complementation of the acs octuple mutant. We complemented the original acs octuple mutant ${ }^{7}$ by expressing genomic versions of the previously-described Arabidopsis ACS8m and ACS11m cDNA sequences ${ }^{7}$ (mutated to prevent targeting by the amiR). Instead of the CaMV $35 \mathrm{~S}$ promoter, we used the native promoter regions of ACS8 and ACS11, $2544 \mathrm{bp}$ and $2755 \mathrm{bp}$ upstream of each start codon, respectively. For each gene, the wild-type genomic sequence spanning the upstream region to the stop codon was amplified in two overlapping fragments using primers shown in Supplementary Table S1. The altered amiR target site was carried by the PCR primers that generated the overlapping fragments. The fragments, proACS8: ASC8m and proACS11:ASC11m, were each separately cloned by Gibson assembly into EcoRI-digested Gateway entry vector pENTR2B and confirmed by DNA sequencing, before their transfer by Gateway cloning into binary vectors pEarlyGate303 and pMDC99 (ABRC), respectively. (SacI/SpeI-digested pMDC99 was first modified by Gibson assembly to carry an OCS terminator sequence that was PCRamplified from pEarleyGate103 using primers shown in Supplementary Table S1). Finally, proACS8:ACS8m was subcloned from pEarlygate303 into pMDC99proACS11:ACS11 $m$ by ligation into BamHI and SbfI sites. The resulting plasmid, carrying both proACS8:ACS8m and proACS11:ACS11m, was stably transformed into the acs octuple mutant as described above. Transgenic plants were confirmed by PCR-genotyping of both the hygromycin resistance gene and the transgene using primers shown in Supplementary Table S1.

Ethylene measurement. Seeds were surface sterilized, then stratified for 3 days at $4{ }^{\circ} \mathrm{C}$, then grown on a sterile filter paper on top of $1 \mathrm{X}$ MS medium ( $0.8 \%$ agar) for 9 days under light $(16 \mathrm{~h} / 8 \mathrm{~h})$ at $22^{\circ} \mathrm{C}$. Approximately 20 seedlings of each genotype (six biological replicates per genotype) were then placed in $300 \mu \mathrm{L}$ liquid MS medium in 4-mL vials fitted with septa-containing airtight caps (Fisher, cat\# 03 391-22). After incubating the vials under light $(16 \mathrm{~h} / 8 \mathrm{~h})$ at $22^{\circ} \mathrm{C}$ for $48 \mathrm{~h}$, we assayed the ethylene concentration in the vial headspace by gas chromatography (Shimadzu GC-2010 Plus). Seedlings were then removed from the vials, blotted briefly on a kimwipe, then weighed. We confirmed there is no ethylene peak for medium in the vial without seedlings. Transgenic lines were homozygous.

Ovule fate determination. Plants of each genotype were grown side-by-side under the same conditions. Siliques at the mature green stage ( $7-10$ days post-pollination) were collected from the middle of the main stem, excluding the first 4-5 siliques and the last several siliques. Siliques were placed on double-side tape and the ovary walls were removed using a 27.5-gauge needle under a dissection microscope as described ${ }^{59}$. Ovule fates were scored in the $T_{1}$ generation for the new acs octuple mutants, since the amiRNA responsible for generating the octuple mutant was dominant. Fertilized ovules that developed normal-looking embryos were scored as "normal embryos". Embryos that were pale green, white, or brown and somewhat flattened were scored as "aborted embryos". Unfertilized ovules, which were very small and wrinkled, were scored as "unfertilized ovules". Images were obtained using a stereoscopic zoom microscope (Nikon SMZ1000).
Reciprocal crosses and pollination time course. For reciprocal crosses in Supplementary Fig. 3, we hand-crossed wild-type pistils using acs octuple mutant pollen, and vice versa. As controls, each genotype was hand-pollinated to itself. For subsequent reciprocal crosses in Fig. 2 , the acs hextuple mutant (female) was crossed with the original acs octuple mutant ${ }^{7}$ (male) to generate $\mathrm{F}_{1}$ plants homozygous for the acs hextuple knockout insertions and hemizygous for the amiR transgene $^{7}$ (responsible for creating the acs octuple mutant in the acs hextuple background). Reciprocal crosses were then carried out between the $F_{1}$ and wild type, and the resulting progeny were genotyped for amiR using primers listed in Supplementary Table S1 to score segregation of the gentamicin resistance gene carried by the $a m i R$ transgene. Inheritance of the $a m i R$ by half the progeny indicated the absence of a gametophytic defect. (A significant reduction in $a m i R$ transmission would have indicated a gametophytic defect). For the pollination time course, flowers were emasculated at stage $12 \mathrm{~b}^{23}$, then hand pollinated with wildtype pollen after 24,36 , and $48 \mathrm{~h}$.

Examination of flower/ovule morphology. Plants of each genotype were grown side-by-side under the same conditions. Flowers at stage $12 \mathrm{~b}$ (pre-pollination) ${ }^{23}$ and stage 14 (post-pollination) ${ }^{23}$ were collected from the middle of the main stem, excluding the first four to five flowers and the last several flowers. Some or all of the outer organs were removed. To examine ovule morphology, stage $12 \mathrm{~b}$ flowers were emasculated and pistils were dissected two days later as described above for ovule fate determination. Images were obtained using a stereoscopic zoom microscope (Nikon SMZ1000).

Pollen tube visualization using proLAT52:GUS. Wild-type and acs octuple mutant flowers were emasculated at floral stage $12 \mathrm{~b}^{23}$, then hand-pollinated $24 \mathrm{~h}$ later using wild-type pollen expressing proLAT52:GUS. For GUS staining, pistils were harvested $22 \mathrm{~h}$ after pollination, and ovary walls were removed as described above for ovule fate determination. Dissected pistils were immediately placed in $80 \%$ acetone for $4 \mathrm{~h}$ then incubated in GUS staining solution $(5 \mathrm{mM}$ potassium ferrocyanide, $5 \mathrm{mM}$ potassium ferricyanide, $50 \mathrm{mM} \mathrm{NaPO}_{4}, \mathrm{pH} 7,0.5 \mathrm{mg} / \mathrm{mL}$ bromo-4-chloro-3-indolyl- $\beta$-D-glucuronic acid) at $37^{\circ} \mathrm{C}$ overnight in the dark in a humid chamber. The GUS stain was visualized using a Zeiss Axioskop 50 microscope, and images were captured using a Sony ILCE-7RM3 camera at $\times 10$ magnification. Composite images were created by pairwise stitching of individual images using Photoshop CS5 (Adobe).

Pollen tubes staining with aniline blue. Flowers were emasculated at stage $12 \mathrm{~b}^{11}$ and the pistils were hand-pollinated $24 \mathrm{~h}$ later. Pistils were detached $18 \mathrm{~h}$ after pollination and fixed in ethanol:acetic acid (3:1) for at least $2 \mathrm{~h}$ at room temperature, then rehydrated in an ethanol series $(70 \% \mathrm{EtOH}, 50 \% \mathrm{EtOH}, 30 \% \mathrm{EtOH}$, then $\mathrm{ddH}_{2} \mathrm{O}$ for $10 \mathrm{~min}$ each). The pistils were then incubated in $8 \mathrm{M} \mathrm{NaOH}$ overnight at room temperature, washed with distilled water for $10 \mathrm{~min}$, then stained overnight at room temperature with decolorized aniline blue $0.1 \%(\mathrm{w} / \mathrm{v})$ in the dark as described ${ }^{60}$. Decolorized aniline blue solution was made by preparing $0.1 \%(\mathrm{w} / \mathrm{v})$ aniline blue in $108 \mathrm{mM} \mathrm{K}_{3} \mathrm{PO}_{4}(\mathrm{pH} \sim 11)$. After storage at $4{ }^{\circ} \mathrm{C}$ overnight, the solution was filtered through filter paper lined funnel with a teaspoon of active carbon powder. Pistils were mounted on slides in $80 \mu \mathrm{L}$ aniline blue stain solution with a cover slip, then imaged using a DeltaVision Elite Deconvolution/ TIRF microscope with a 10x objective using an excitation wavelength of $420-490$ $\mathrm{nm}$ and emission wavelength of $510 \mathrm{~nm}$. Composite images were created using the ImageJ pairwise stitching plugin ${ }^{61}$ to join individual images. We examined at least 15 pistils per genotype.

Semi-in vivo pollen tube guidance assay. The semi-in vivo pollen tube guidance assay was performed as described ${ }^{24}$. In brief, after hand-pollination, the pistil was cut horizontally at the junction of style and ovary, then placed on pollen tube growth medium (18\% Sucrose; $0.01 \%$ Boric acid; $1 \mathrm{mM} \mathrm{CaCl} ; 1 \mathrm{mM} \mathrm{Ca}\left(\mathrm{NO}_{3}\right)_{2} ; 1$ $\mathrm{mM} \mathrm{MgSO}_{4} ; 0.5 \%$ Noble agar (Difco)) in a small petri dish (35 $\mathrm{mm}$ diameter). Next, ovules were excised from pistils and immediately placed on the medium with micropylar ends facing the cut style. For "ovule competition", wild-type and mutant ovules (harvested $24 \mathrm{~h}$ after emasculation at floral stage $12 \mathrm{~b}^{23}$ ) were arranged on the left/right or right/left sides with respect to the cut style. Each agar plate (with the lid on) was incubated on a water-soaked paper towel inside a larger (150 $\mathrm{mm}$ diameter) petri dish (with the lid on) in an airtight food storage container that was also lined with a water-soaked paper towel. After $\sim 4 \mathrm{~h}$ at room temperature, pollen tubes were viewed under a Leica M205 FA microscope and images captured using a SONY SLT-A55V camera.

Evaluation of micropylar guidance of pollen tubes was based on separate regions beneath the cut style. As shown in Supplementary Fig. 6, we defined the left and right ovule regions as the areas extending from the center of the cut style to between the micropyle of the leftmost or rightmost ovule and the micropyle of the central-most ovule on the left or right, respectively. All the pollen tube tips in the left region (or right) were scored as turning to the ovules on the left (or right). Pollen tube tips between the left and right regions were regarded as turning to neither region. Pollen tube tips at either the far left or far right of the defined regions were considered out of range and were not included in the total pollen tube number. 
For ACC pretreatment, we dissected pistils $24 \mathrm{~h}$ after emasculation at floral stage $12 b^{23}$ as described above for ovule fate determination, and to each dissected pistil we added $1.0 \mu \mathrm{L} 1 \mu \mathrm{M}$ ACC, which covered all the ovules in the pistil. Immediately after absorbing the ACC solution (5-10 min), ovules were arranged on the agar medium taking care not to let the ovules dry out. Pretreatment with 1.0 $\mu \mathrm{L}$ sterile $\mathrm{ddH}_{2} \mathrm{O}$ was used as a control. For ethylene pretreatment, the dissected pistil was transferred to semi-in vivo agar medium in a small petri dish, and the dish (with its lid partially off) was placed on a water-soaked paper towel in an airtight 1.0 L Mason jar fitted with a septum in its lid. Ethylene gas was injected through the septum to provide a final concentration of $10 \mathrm{ppm}$ in the jar. Injection of ambient air was the control. After $3 \mathrm{~h}$ at room temperature, the ovules were placed in the semi-in vivo assay as above. For continuous ethylene treatment, we conducted the entire assay under ethylene by placing the agar plate containing the stigma-style and ovules into the Mason jar, injecting ethylene to a final concentration of $10 \mathrm{ppm}$ and incubating for $\sim 4 \mathrm{~h}$ before imaging.

To test whether ACC functions as a pollen tube chemoattractant, we used $6 \%$ Sepharose beads $(200-300 \mu \mathrm{m})$ (Colloidal Science Solutions) in place of ovules. Beads were prewashed five times ( 5 min each) with sterile $\mathrm{ddH}_{2} \mathrm{O}$, then immersed in either $1 \mu \mathrm{M} \mathrm{ACC}$ or $\mathrm{ddH}_{2} \mathrm{O}$ for $30 \mathrm{~min}$ prior to positioning a bead on the agar medium on either side below, and equidistant to, the cut style.

qPCR of LURE1s. RNA was extracted from stage 12b flowers of the acs hextuple and octuple mutants using SpectrumTM Plant Total RNA Kit (Sigma), then reverse-transcribed using iScript ${ }^{\text {mi }}$ cDNA Synthesis Kit (Bio-Rad). qPCR was performed on a Bio-Rad CFX96 system using iTaq ${ }^{\mathrm{TM}}$ Universal SYBR Green Supermix (Bio-Rad), using a single primer pair (Supplementary Table S1) to amplify a conserved sequence in all five LURE1.1-1.5 genes. Relative expression was calculated using the $2^{-\Delta \Delta C T}$ method $^{62}$ normalizing to TUBULIN5 (AT1G20010.1) using primers shown in ref. ${ }^{20}$. We carried out 12 biological replicates per genotype, and three technical replicates per biological replicate.

proLURE1.2:LURE1.2-eGFP construct and localization. To generate the plasmid backbone, binary vector pEarleyGate103 (ABRC) was digested with MluI and PacI, which removed the CaMV $35 \mathrm{~S}$ promoter and GFP sequence. The following DNA fragments were PCR-amplified using primers shown in Supplementary Table S1: (a) a fragment spanning the MluI site to just before the start of the CaMV $35 \mathrm{~S}$ promoter amplified from pEarleyGate103, (b) a 658-bp fragment containing the LURE1.2 promoter/coding region amplified from wild-type Arabidopsis genomic DNA, and (c) the eGFP coding sequence amplified from plasmid pK7FWG $2^{63}$ (ABRC). After gel-purification, the three fragments and plasmid backbone were combined by Gibson assembly.

We next replaced the Basta-resistance gene in the resulting plasmid with a hygromycin-resistance gene, as the acs octuple mutant is already Basta-resistant We removed the Basta-resistance gene by KpnI and ClaI, and a hygromycinresistance gene fragment was PCR-amplified from pMDC99 using primers listed in Supplementary Table S1. Fragments were combined by Gibson assembly creating the final plasmid proLURE1.2:LURE1.2-eGFP. Plants were transformed and homozygous lines obtained as described for the new acs octuple mutant alleles.

For eGFP visualization, flowers of hygromycin-resistant $T_{2}$ or homozygous $T_{3}$ plants were emasculated at floral stage $12 \mathrm{c}^{23}$, and pistils were harvested $24 \mathrm{~h}$ later as described ${ }^{64}$. Pistils were dissected and mounted on microscope slides as described ${ }^{59}$. For pretreatment with ACC $(1 \mu \mathrm{M})$, ethylene (10 ppm), or A23187 (10 $\mu \mathrm{M}$ ), ovules were treated as described for the semi-in vivo pollen tube guidance assay, except they were incubated for $2.5-3.5 \mathrm{~h}$. After treatment, pistils were transferred to slides and mounted with Fluoromount $-G^{\oplus}$ (SouthernBiotech) and coverslips. eGFP fluorescence was visualized under a Zeiss LSM 710 laser scanning confocal microscope coupled with $\times 40$ oil-immersion objective, exciting eGFP with a 488 -nm $\operatorname{argon}$ laser. The $Z$-axis focus was adjusted during observation.

Knockout of ein2 in the acs octuple mutant. Binary vector V3 ${ }^{20}$, carrying eGFPtagged oleosin to screen for transformants, was digested with SpeI/EcoRI and ligated to a $6275 \mathrm{bp} \mathrm{SpeI/EcoRI} \mathrm{fragment} \mathrm{(containing} \mathrm{the} \mathrm{Ec1.2enEC1.1} \mathrm{promoter,}$ zCas9 and RBCS terminator) of plasmid p425-pHEE401E ${ }^{65}$. The resulting plasmid was digested with KpnI/SpeI and combined by Gibson assembly with a Gateway destination cassette (PCR-amplified from a modified pHEE401E ${ }^{20}$ template using primers shown in Supplementary Table S1) to create destination vector p568.

To create the EIN2 CRISPR/Cas9 transformation vector, we generated two guide RNAs shown in Supplementary Table S1) that targeted different locations in the EIN2 sequence. Four overlapping fragments were PCR-amplified from pCAMBIA1302Cas9-sgRNA ${ }^{66}$ using the primers shown in Supplementary Table S1. The fragments were cloned by Gibson assembly into Sall/XhoI-digested pENTR2B (Invitrogen), creating an EIN2 guide plasmid (U6-26pro:gRNA1-scaffold-U6-26 terminator and U6-26pro:gRNA2-scaffold-U6-26 terminator), which we confirmed by DNA sequencing. The construct was transferred into p568 by Gateway cloning.

The final construct was transformed into the acs octuple mutant as described above, except the Agrobacterium carried the pSoup ${ }^{67}$ helper plasmid. $\mathrm{T}_{1}$ seeds were identified based on seed coat fluorescence, and PCR-based genotyping was used to identify deletions in the EIN2 sequence using primers shown in Supplementary Table S1. In the $\mathrm{T}_{2}$ generation, we obtained one plant that was heterozygous for a 407-bp deletion, and we identified a homozygous mutant via PCR-screening of $\mathrm{T}_{3}$ progeny that lacked zCas9 (the seeds were non-fluorescent). The homozygous line was confirmed by assaying for absence of the ethylene response in dark-grown seedlings ${ }^{1}$, and then transformed with proLURE1.2:LURE1.2-eGFP as described above. $T_{1}$ plants were analyzed.

proACS8:GUS. The ACS8:GUS reporter gene ${ }^{25}$ (ABRC, \#CD3-721) was stably transformed into wild-type Arabidopsis as above, and transformants were selected on gentamycin $(100 \mu \mathrm{g} / \mathrm{mL})$. Stage 12b flowers were emasculated and ovary walls were removed $24 \mathrm{~h}$ later as described ${ }^{59}$. Dissected pistils were stained and imaged for GUS activity as described for proLAT52:GUS. We analyzed ten $\mathrm{T}_{1}$ plants, and six showed the same expression pattern shown in Supplementary Fig. $7 \mathrm{~g}$.

Patch-clamp of Arabidopsis protoplasts. Roots were collected from 14-day-old Arabidopsis seedlings grown on MS agar medium under long day $(16 \mathrm{~h})$ at $19^{\circ} \mathrm{C}$ with the plates incubated vertically. The root digestion solution, which was prepared $12 \mathrm{~h}$ prior to use and stored at $4{ }^{\circ} \mathrm{C}$, consisted of $1.5 \% \mathrm{w} / \mathrm{v}$ cellulase $\mathrm{R} 10$ (Omozuka), $0.4 \% \mathrm{w} / \mathrm{v}$ pectolyase Y23 (Omozuka), 1.5\% cellulozyme (Fisher), $0.4 \mathrm{M}$ D-Mannitol, $20 \mathrm{mM}$ MES (pH 5.7, Tris $\mathrm{HCl}$ ) and $20 \mathrm{mM} \mathrm{KCl}$ ). The solution was heated for $10 \mathrm{~min}$ at $55^{\circ} \mathrm{C}$, and then $\mathrm{BSA}$ and $\mathrm{CaCl}_{2}$ were added to a final concentration of $0.1 \% \mathrm{BSA}$ and $10 \mathrm{mM} \mathrm{CaCl}_{2}$. Roots were incubated in this solution for $1 \mathrm{~h}$ at $30^{\circ} \mathrm{C}$ with agitation. Protoplasts were centrifuged and washed in two successive rounds with patch-clamp bathing solution $\left(10 \mathrm{mM} \mathrm{NaCl}, 20 \mathrm{mM} \mathrm{CaCl}_{2}\right.$, $90 \mathrm{mM}$ NMDG-Cl and $10 \mathrm{mM}$ Bis-Tris propane, $\mathrm{pH} 6.5$ (MES)). The pipette solution contained $140 \mathrm{mM} \mathrm{NaCl}, 3 \mathrm{mM} \mathrm{MgCl}, 5 \mathrm{mM}$ EGTA, and $10 \mathrm{mM}$ Bis-Tris

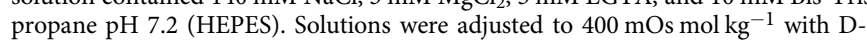
mannitol. Protoplasts were stored on ice up to $3 \mathrm{~h}$ prior to experimentation. Pipettes were pulled with a P97 puller (Sutter Instrument). Their resistance was 10-20 Mohm. Currents were recorded after establishing the whole-cell configuration ${ }^{68}$, filtered at $1-2 \mathrm{kHz}$ with a sampling frequency of $2-4 \mathrm{kHz}$ using an Axopatch $200 \mathrm{~A}$ amplifier, digidata 1200 series interface and Clampfit6 software (Molecular Devices). After entering the whole-cell configuration, we waited $10 \mathrm{~min}$ for the pipette solution to diffuse into the protoplast and the current to stabilize. The voltage protocol consisted of $1.6 \mathrm{~s}$-long pulses from $-140 \mathrm{mV}$ to $+60 \mathrm{mV}$ (20 mV steps) both before, and $5 \mathrm{~min}$ after, adding either $\mathrm{ACC}$ or $\mathrm{GdCl}_{3}$ to the bath solution.

\section{$\mathbf{C a}^{2}+$ imaging in COS-7 cells. COS-7 cells (ATCC and Sigma-Aldrich) were} maintained at $37^{\circ} \mathrm{C}$ and $5 \% \mathrm{CO}_{2}$ in Dulbecco's Modified Eagle's Medium, sup plemented with $5 \%$ fetal bovine serum and $1 \%$ penicillin/streptomycin (Gibco), and transfected at low passage $(P<7)$. COS-7 cells were plated at a density of $50 \%$ confluence in 35-mm diameter dishes and transfected using FugeneHD (Promega) as specified by the supplier. For $\mathrm{Ca}^{2+}$ imaging, pCI $(0.6 \mu \mathrm{g}$ or $1 \mu \mathrm{g})$ or pCIPpGLR1 ${ }^{28}(1 \mu \mathrm{g})$ was co-transfected with pEF1-YC3.6 $(0.5 \mu \mathrm{g})$. COS-7 cells were transferred to new petri dishes $24 \mathrm{~h}$ after transfection (by trypsin treatment) at low density.

For $\mathrm{Ca}^{2+}$ imaging, COS-7 cells expressing YC3.6 were washed in a $\mathrm{Ca}^{2+}$-free solution (1 mM EGTA, $10 \mathrm{mM}$ Bis-Tris propane buffered to $\mathrm{pH} 7.3$ (HEPES) and set to $335 \mathrm{mosmol} \mathrm{kg}{ }^{-1}$ with D-mannitol). Cells were imaged in the $\mathrm{Ca}^{2+}$-free solution for $2.5 \mathrm{~min}$ before the addition of $\mathrm{Ca}^{2+}$ to a final concentration of 14.5 $\mathrm{mM}$. Imaging was performed at room temperature using a DeltaVision Elite Deconvolution/TIRF microscope system (Olympus inverted IX-71) under a $60 \times$ lens (1.2NA UPLSAPO water lens/WD $0.28 \mathrm{~mm}$ ) as described ${ }^{69}$. A xenon lamp from the DeltaVision system was used with a CFP excitation filter (438-424 nm) Two simultaneous emission records were captured: YFP emission $(548-522 \mathrm{~nm})$ and CFP emission (475-424 nm). To minimize bleaching, the laser was set to $2 \%$. YFP and CFP imaging were recorded with $0.3 \mathrm{~s}$ exposure time. Time-lapse acquisition was performed with a sampling interval of $30 \mathrm{~s}$. Images were processed using Image ${ }^{53}$. Ratios were obtained after background subtraction and signal clipping using the "Ratio-plus" plug-in for ImageJ as described ${ }^{69}$. In the ligand screening experiments (Fig. 3d and Supplementary Fig. 8d), values were multiplied by a factor of one hundred. The signal of each channel was averaged in a circle in the middle of the cell (with 100-200 pixel diameter depending on the size of the cell). The YFP/CFP ratio was obtained by dividing the emission recorded for YFP (548-522 nm) by the one recorded for CFP (475-424 nm). No significant bleaching or ratio drift was observed in our experimental conditions. To standardize YC3.6 responses, the baseline values (five points prior to $\mathrm{Ca}^{2+}$ application) were averaged and then subtracted from each measurement recorded. The integrated area under the curve was calculated in SigmaPlot 11.0 (Systat Software Inc) by drawing a bestfit line and using the built in macro "Area Below Curves".

$\mathbf{C a}^{2+}$ imaging in ein2-5 ovules. We crossed a cytosolic GCaMP3 reporter in the wild type (gift from Simon Gilroy, University of Wisconsin, Madison) into the ein2-5 mutant and identified double homozygotes in the $\mathrm{F}_{3}$ based on ethylene insensitivity and kanamycin resistance $(50 \mu \mathrm{g} / \mathrm{mL})$. Flowers were emasculated at Stage $12 \mathrm{c}$, and 24 $\mathrm{h}$ later the ovules were cut from dissected pistils and placed on a thin layer of agar medium created by spreading $100 \mu \mathrm{L}$ of pollen germination medium ${ }^{24}$ over the entire coverslip of a glass bottom petri dish (MatTek, P35G-1.0-14-C)

$\mathrm{Ca}^{2+}$ signals were visualized under a Nikon ECLIPSE microscope, using dry PlanApo lenses of either $20 \times($ NA0.75) or $40 \times($ NA1.0). Wavelength was controlled 
by a Prior Lumen 200 Pro Fluorescence Illumination System, with a GFP filter, and images were captured by a iXON3 ANDOR camera using Micro-Manager 1.4.16. Prior to treatments, we imaged ovules every $10 \mathrm{~s}$ for $2 \mathrm{~min}$. For treatments, $5 \mu \mathrm{L}$ of ACC $(500 \mu \mathrm{M}), \mathrm{CNQX}(50 \mu \mathrm{M})$, or sterile $\mathrm{ddH}_{2} \mathrm{O}$ was added to the surface of the germination medium covering all of the ovules, and the microscope was immediately refocused. We then imaged the ovules every $10 \mathrm{~s}$ for $10 \mathrm{~min}$. For the CNQX-treated samples, we subsequently added ACC $(500 \mu \mathrm{M})$, and continued imaging every $10 \mathrm{~s}$ for $10 \mathrm{~min}$. We imaged 10 ovules treated with ACC, 11 ovules treated with ACC after CNQX pretreatment, and 13 ovules treated with $\mathrm{ddH}_{2} \mathrm{O}$.

We quantified $\mathrm{Ca}^{2+}$ signal intensity using Image ${ }^{70}$. We selected the entire ovule as the "region of interest (ROI)" and quantified ROI fluorescence by selecting "Mean" under "Set measurements". In the ROI menu, we chose "more" and "multi measure" to obtain the mean pixel value across all time points. We subtracted background fluorescence from each ovule pixel value. Background was determined by measuring the pixel value of three blank areas, each of similar size and shape as an ovule, and then averaging the values. Further processing was made by normalizing sequences to background values, and applying the ICA LUT for visualization.

The relative $\mathrm{Ca}^{2+}$ variance ratio time course (Fig. $\left.4 \mathrm{~b}\right)$ was calculated as $\left(F_{n}-\right.$ $\left.F_{n-1}\right) / F_{n-1}$ where $F_{\mathrm{n}}$ is the fluorescence value at time point $n$, and $F_{n-1}$ is the value at one time point prior. The percentage of $\mathrm{Ca}^{2+}$ signal increase (Fig. 4c) was calculated as $\left(F_{\max }-F_{0}\right) / F_{0}$ where $F_{0}$ is the value for the first frame in the time series and $F_{\max }$ is the maximum value in the series.

Statistical analyses. All measurements were taken from distinct samples. All statistical analyses were performed using Prism (8.0.1, GraphPad) or SigmaPlot (v11.0, Systat Software Inc). For comparisons between two groups, we used the $t$ test (two tailed) for populations having a normal (Gaussian) distribution when both groups had the same SD. When the two populations did not have the same $\mathrm{SD}$, we used the $t$-test with Welch's correction (two tailed). For populations lacking a normal (Gaussian) distribution, we used two nonparamatric tests: the Mann-Whitney test to compare ranks and the Kolmogorov-Smirnov test to compare the cumulative distribution. For comparisons among multiple groups, we used one-way ANOVA with Tukey's HSD post hoc test for populations that have normality of residuals and the same SD. When SDs were unequal, we used the Welch ANOVA tests with Tamhane's T2 post hoc test. For data lacking a normal distribution of residuals, we used the nonparametric Kruskal-Wallis test with Dunn's post hoc test. For Fig. 3a, b and Supplementary Figs. 8a, b, we used ANOVA. For Fig. 3d and Supplementary Fig. 8d, we used a Dunnett's test for comparison of multiple experimental treatments to a single control. For Fig. 3c, we used the RM two-way ANOVA with Geisser-Greenhouse correction with Tukey's HSD post hoc test to compare the mean value for each of the whole curve.

Reporting summary. Further information on research design is available in the Nature Research Reporting Summary linked to this article.

\section{Data availability}

Source data are provided with this paper as a Source Data file. Other data and biological materials are available from the corresponding authors upon reasonable request. Source data are provided with this paper.

Received: 26 April 2020; Accepted: 20 July 2020;

Published online: 14 August 2020

\section{References}

1. Abeles, F. B. M., Morgan, P. W. \& Saltveit, M. E. Ethylene in Plant Biology (Academic, 1992).

2. Adams, D. O. \& Yang, S. F. Ethylene biosynthesis-identification of 1aminocyclopropane-1-carboxylic acid as an intermediate in the conversion of methionine to ethylene. Proc. Natl Acad. Sci. U.S.A. 76, 170-174 (1979).

3. Vanderstraeten, L., Depaepe, T., Bertrand, S. \& Van Der Straeten, D. The ethylene precursor ACC affects early vegetative development independently of ethylene signaling. Front. Plant Sci. 10, 1591 (2019).

4. Tsang, D. L., Edmond, C., Harrington, J. L. \& Nuhse, T. S. Cell wall integrity controls root elongation via a general 1-aminocyclopropane-1-carboxylic aciddependent, ethylene-independent pathway. Plant Physiol. 156, 596-604 (2011).

5. Xu, S. L., Rahman, A., Baskin, T. I. \& Kieber, J. J. Two leucine-rich repeat receptor kinases mediate signaling, linking cell wall biosynthesis and ACC synthase in Arabidopsis. Plant Cell 20, 3065-3079 (2008).

6. Yin, J. et al. Aminocyclopropane-1-carboxylic acid is a key regulator of guard mother cell terminal division in Arabidopsis thaliana. J. Exp. Bot. 70, 897-907 (2019).
7. Tsuchisaka, A. et al. A combinatorial interplay among the 1aminocyclopropane-1-carboxylate isoforms regulates ethylene biosynthesis in Arabidopsis thaliana. Genetics 183, 979-1003 (2009).

8. Johnson, M. A., Harper, J. F. \& Palanivelu, R. A fruitful journey: pollen tube navigation from germination to fertilization. Annu. Rev. Plant Biol. 70, 809-837 (2019).

9. Zhong, S. et al. Cysteine-rich peptides promote interspecific genetic isolation in Arabidopsis. Science 364, eaau9564 (2019).

10. Stewman, S. F. et al. Mechanistic insights from a quantitative analysis of pollen tube guidance. BMC Plant Biol. 10, 32 (2010).

11. Feijó, J. A. The mathematics of sexual attraction. J. Biol. 9, 18 (2010).

12. Ge, L. L., Tian, H. Q. \& Russell, S. D. Calcium function and distribution during fertilization in angiosperms. Am. J. Bot. 94, 1046-1060 (2007).

13. Ngo, Q. A., Vogler, H., Lituie, D. S., Nestorova, A. \& Grossniklaus, U. A calcium dialog mediated by the FERONIA signal transduction pathway controls plant sperm delivery. Dev. Cell. 29, 491-500 (2014).

14. Denninger, P. et al. Male-female communication triggers calcium signatures during fertilization in Arabidopsis. Nat. Commun. 5, 4645 (2014).

15. Hamamura, Y. et al. Live imaging of calcium spikes during double fertilization in Arabidopsis. Nat. Commun. 5, 4722 (2014).

16. Wudick, M. M., Michard, E., Nunes, C. O. \& Feijó, J. A. Comparing plant and animal glutamate receptors: common traits but different fates? J. Exp. Bot. 69, 4151-4163 (2018).

17. Michard, E. et al. GLUTAMATE RECEPTOR-LIKE genes form $\mathrm{Ca}^{2+}$ channels in pollen tubes and are regulated by pistil D-serine. Science 332, 434-437 (2011).

18. Ortiz-Ramirez, C. et al. GLUTAMATE RECEPTOR-LIKE channels are essential for chemotaxis and reproduction in mosses. Nature 549, 91-95 (2017).

19. Michard, E., Simon, A. A., Tavares, B., Wudick, M. M. \& Feijó, J. A. Signaling with ions: the keystone for apical cell growth and morphogenesis in pollen tubes. Plant Physiol. 173, 91-111 (2017).

20. Wudick, M. M. et al. CORNICHON sorting and regulation of GLR channels underlie pollen tube $\mathrm{Ca}^{2+}$ homeostasis. Science 360, 533-536 (2018).

21. Okuda, S. et al. Defensin-like polypeptide LUREs are pollen tube attractants secreted from synergid cells. Nature 458, 357-U122 (2009).

22. Takeuchi, H. \& Higashiyama, T. A species-specific cluster of defensin-like genes encodes diffusible pollen tube attractants in Arabidopsis. PLoS Biol. 10, e1001449 (2012).

23. Christensen, C. A., King, E. J., Jordan, J. R. \& Drews, G. N. Megagametogenesis in Arabidopsis wild type and the Gf mutant. Sex. Plant Reprod. 10, 49-64 (1997).

24. Palanivelu, R. \& Preuss, D. Distinct short-range ovule signals attract or repel Arabidopsis thaliana pollen tubes in vitro. BMC Plant Biol. 6, 7 (2006).

25. Tsuchisaka, A. \& Theologis, A. Unique and overlapping expression patterns among the Arabidopsis 1-amino-cyclopropane-1-carboxylate synthase gene family members. Plant Physiol. 136, 2982-3000 (2004).

26. Mizuta, Y. \& Higashiyama, T. Chemical signaling for pollen tube guidance at a glance. J. Cell Sci. 131, jcs208447 (2018).

27. Völz, R., Heydlauff, J., Ripper, D., von Lyncker, L. \& Gross-Hardt, R. Ethylene signaling is required for synergid degeneration and the establishment of a pollen tube block. Dev. Cell 25, 310-316 (2013).

28. De Martinis, D. \& Mariani, C. Silencing gene expression of the ethyleneforming enzyme results in a reversible inhibition of ovule development in transgenic tobacco plants. Plant Cell 11, 1061-1071 (1999).

29. Inanobe, A., Furukawa, H. \& Gouaux, E. Mechanism of partial agonist action at the NR1 subunit of NMDA receptors. Neuron 47, 71-84 (2005).

30. Yao, Y., Harrison, C. B., Freddolino, P. L., Schulten, K. \& Mayer, M. L. Molecular mechanism of ligand recognition by NR3 subtype glutamate receptors. EMBO J. 27, 2158-2170 (2008).

31. Kristensen, A. S. et al. Pharmacology and structural analysis of ligand binding to the orthosteric site of glutamate-like GluD2 receptors. Mol. Pharmacol. 89 , 253-262 (2016).

32. Traynelis, S. F. et al. Glutamate receptor ion channels: structure, regulation, and function. Pharmacol. Rev. 62, 405-496 (2010).

33. Vincill, E. D., Bieck, A. M. \& Spalding, E. P. $\mathrm{Ca}^{2+}$ conduction by an amino acid-gated ion channel related to glutamate receptors. Plant Physiol. 159, 40-46 (2012).

34. Tapken, D. et al. A plant homolog of animal glutamate receptors is an ion channel gated by multiple hydrophobic amino acids. Sci. Signal. 6, ra47 (2013).

35. Alfieri, A. et al. The structural bases for agonist diversity in an Arabidopsis thaliana glutamate receptor-like channel. Proc. Natl Acad. Sci. U.S.A. 117, 752-760 (2020).

36. Nagai, T., Yamada, S., Tominaga, T., Ichikawa, M. \& Miyawaki, A. Expanded dynamic range of fluorescent indicators for $\mathrm{Ca}^{2+}$ by circularly permuted yellow fluorescent proteins. Proc. Natl Acad. Sci. U.S.A. 101, 10554-10559 (2004).

37. Nakai, J., Ohkura, M. \& Imoto, K. A high signal-to-noise $\mathrm{Ca}(2+)$ probe composed of a single green fluorescent protein. Nat. Biotechnol. 19, 137-141 (2001). 
38. Yabut et al. Genetic deletion of mast cell serotonin synthesis prevents the development of obesity and insulin resistance. Nat. Commun. 11, 463 (2020).

39. Diao, M., Qu, X. \& Huang, S. Calcium imaging in Arabidopsis pollen cells using G-CaMP5. J. Int. Plant Biol. 60, 897-906 (2018).

40. Antoine, A. F., Faure, J. E., Dumas, C. \& Feijo, J. A. Differential contribution of cytoplasmic $\mathrm{Ca}^{2+}$ and $\mathrm{Ca}^{2+}$ influx to gamete fusion and egg activation in maize. Nat. Cell Biol. 3, 1120-1123 (2001).

41. Malho, R. \& Trewavas, A. J. Localized apical increases of cytosolic free calcium control pollen tube orientation. Plant Cell 8, 1935-1949 (1996).

42. Boavida, L. C., Borges, F., Becker, J. D. \& Feijó, J. Whole genome analysis of gene expression reveals coordinated activation of signaling and metabolic pathways during pollen-pistil interactions in Arabidopsis. Plant Physiol. 155, 2066-2080 (2011).

43. Vanderstraeten, L. \& Van der Straeten, D. Accumulation and transport of 1aminocyclopropane-1-carboxylic acid (ACC) in plants: current status, considerations for future research and agronomic applications. Front. Plant Sci. 8, 38 (2017).

44. Osborne, D. J., Walters, J., Milborrow, B. V., Norville, A. \& Stange, L. M. C. Evidence for a non-ACC ethylene biosynthesis pathway in lower plants. Phytochemistry 42, 51-60 (1996).

45. Li, F.-W. et al. Fern genomes elucidate land plant evolution and cyanobacterial symbioses. Nat. Plants 4, 460-472 (2018)

46. VanWent, J. L. \& Willemse, M. T. M. Fertilization. In Embryology of Angiosperms (ed. Johri, B. M.) 273-317 (Springer, 1984).

47. Linskens, H. F. \& Spanjers, A. W. Changes of the electrical potential in the transmitting tissue of Petunia-styles after cross and self-pollination. Incomp Newslett. 3, 81-85 (1973).

48. Spanjers, A. W. Voltage variation in Lilium longiflorum pistils induced by pollination. Experientia 34, 36-37 (1978).

49. Spanjers, A. W. Bioelectric potential changes in the style of Lilium longiflorum Thunb. after self- and cross-pollination of the stigma. Planta 153, 1-5 (1981).

50. Mousavi, S. A., Chauvin, A., Pascaud, F., Kellenberg, S. \& Farmer, E. E. GLUTAMATE RECEPTOR-LIKE genes mediate leaf-to-leaf wound signaling. Nature 500, 422-426 (2013).

51. Toyota, M. et al. Glutamate triggers long-distance, calcium-based plant defense signaling. Science 361, 1112-1115 (2018).

52. Leydon, A. R. et al. Pollen tube discharge completes the process of synergid degeneration that is initiated by pollen tube-synergid interaction in Arabidopsis. Plant Physiol. 169, 485-496 (2015).

53. Schindelin, J. et al. Fiji: an open-source platform for biological-image analysis. Nat. Methods 9, 676-682 (2012).

54. Schwab, R., Ossowski, S., Riester, M., Warthmann, N. \& Weigel, D. Highly specific gene silencing by artificial microRNAs in Arabidopsis. Plant Cell 18, 1121-1133 (2006).

55. Curtis, M. D. \& Grossniklaus, U. A gateway cloning vector set for highthroughput functional analysis of genes in planta. Plant Physiol. 133, 462-469 (2003).

56. Clough, S. J. \& Bent, A. F. Floral dip: a simplified method for Agrobacterium-mediated transformation of Arabidopsis thaliana. Plant J. 16, 735-743 (1998).

57. Koncz, C., Nemeth, K., Redei, G. P. \& Schell, J. T-DNA insertional mutagenesis in Arabidopsis. Plant Mol. Biol. 20, 963-976 (1992).

58. Harrison, S. J. et al. A rapid and robust method of identifying transformed Arabidopsis thaliana seedlings following floral dip transformation. Plant Methods 2, 19 (2006).

59. Johnson, M. A. \& Kost, B. in Plant Developmental Biology. Methods in Molecular Biology (Methods and Protocols), vol 655 (eds Hennig, L. \& Köhler, C.) 155-176 (Humana, 2010).

60. Mori, T., Kuroiwa, H., Higashiyama, T. \& Kuroiwa, T. Generative cell specific 1 is essential for angiosperm fertilization. Nat. Cell Biol. 8, 64-71 (2006).

61. Preibisch, S., Saalfeld, S. \& Tomancak, P. Globally optimal stitching of tiled 3D microscopic image acquisitions. Bioinformatics 25, 1463-1465 (2009).

62. Livak, K. J. \& Schmittgen, T. D. Analysis of relative gene expression data using real-time quantitative PCR and the 2(-Delta Delta C(T)) method. Methods 25, 402-408 (2001).

63. Karimi, M., Inzé, D. \& Depicker, A. Gateway vectors for Agrobacteriummediated plant transformation. Trends Plant Sci. 7, 193-195 (2002).

64. Steffen, J. G., Kang, I. H., Macfarlane, J. \& Drews, G. N. Identification of genes expressed in the Arabidopsis female gametophyte. Plant J. 51, 281-292 (2007).

65. Wang, Z.-P. et al. Egg cell-specific promoter-controlled CRISPR/Cas9 efficiently generates homozygous mutants for multiple target genes in Arabidopsis in a single generation. Genome Biol. 16, 144 (2015).
66. Jiang, W. et al. Demonstration of CRISPR/Cas9/sgRNA-mediated targeted gene modification in Arabidopsis, tobacco, sorghum and rice. Nucleic Acids Res. 41, e188 (2013)

67. Hellens, R. P., Edwards, E. A., Leyland, N. R., Bean, S. \& Mullineaux, P. M. pGreen: a versatile and flexible binary $\mathrm{Ti}$ vector for Agrobacterium-mediated plant transformation". Plant Mol. Biol. 42, 819-832 (2000).

68. Hamill, O. P., Marty, A., Neher, E., Sakmann, B. \& Sigworth, F. J. Improved patch-clamp techniques for high-resolution current recording from cells and cell-free membrane patches. Pflügers Arch. 391, 85-100 (1981).

69. Palmer, A. E. \& Tsien, R. Y. Measuring calcium signaling using genetically targetable fluorescent indicators. Nat. Protoc. 1, 1057-1065 (2006).

70. Shankar, S., Calvert, M. E. K. \& Yew, J. Y. Measuring physiological responses of Drosophila sensory neurons to lipid pheromones using live calcium imaging. J. Vis. Exp. 110, e53392 https://doi.org/10.3791/53392 (2016)

\section{Acknowledgements}

We thank Dr. Edward Farmer for $g l r$ double and quadruple mutants, Prof. Jörg Kudla for pEF1-YC3.6, Dr. Ravishankar Palanivelu for the proLat52:GUS line, Dr. Simon Gilroy for the GCaMP3 line, Dr. Bram Van de Poel for the eto1, eol1, eol2 mutant, Drs. Charles Delwiche, Heven Sze, and Zhongchi Liu for use of their microscopes, Dr. Norma Andrews for sharing her cell culture facilities, Amy Beavan of the Imaging Core Facility (University of Maryland, College Park) for confocal microscopy assistance, and Drs. Palanivelu, Alice Cheung and Stephen Mount for advice. This work was supported by an NSF MCB-1714993 grant (to C.C. and J.A.F.), NSF MCB-1616437, NSF MCB-1930165 and NIH-GM 1R01GM131043-01 grants (to J.A.F.), and graduate student fellowships from the China Scholarship Council (to W.M. and D.L.). CC was partially supported by the Maryland Agricultural Experiment Station.

\section{Author contributions}

All authors were involved in aspects of the experimental design. C.C. and J.A.F. directed the project. W.M. carried out or assisted in all experiments, except for the GLR experiments performed by E.M. (patch-clamp) and A.A.S. $\left(\mathrm{Ca}^{2+}\right.$ imaging in COS-7 cells). W.M. and Y.T.K. conducted experiments relating to LURE1.2-eGFP localization. Y.T.K., W.M., and D.L. generated and analyzed the new octuple mutants and the complemented mutant. W.M. carried out the semi-in vivo assay and qPCR analyses with assistance from D.L. W.M. carried out calcium imaging in ovules with direction from J.A. F. using a GCaMP3 line generated by Y.T.K. M.M.W. performed reciprocal crosses with the $a m i R$ hemizygote. W.M., D.L., Y.T.K., and M.A.L. generated plasmid constructs. C.C. wrote the paper with assistance from J.A.F., W.M., and E.M. Y.T.K. and W.M. assembled the figures. All authors reviewed and commented on the manuscript.

\section{Competing interests}

The authors declare no competing interests.

\section{Additional information}

Supplementary information is available for this paper at https://doi.org/10.1038/s41467020-17819-9.

Correspondence and requests for materials should be addressed to J.A.F. or C.C.

Peer review information Nature Communications thanks Sergey Shabala and the other, anonymous reviewer(s) for their contribution to the peer review of this work.

Reprints and permission information is available at http://www.nature.com/reprints

Publisher's note Springer Nature remains neutral with regard to jurisdictional claims in published maps and institutional affiliations.

Open Access This article is licensed under a Creative Commons Attribution 4.0 International License, which permits use, sharing, adaptation, distribution and reproduction in any medium or format, as long as you give appropriate credit to the original author(s) and the source, provide a link to the Creative Commons license, and indicate if changes were made. The images or other third party material in this article are included in the article's Creative Commons license, unless indicated otherwise in a credit line to the material. If material is not included in the article's Creative Commons license and your intended use is not permitted by statutory regulation or exceeds the permitted use, you will need to obtain permission directly from the copyright holder. To view a copy of this license, visit http://creativecommons.org/ licenses/by/4.0/.

(C) The Author(s) 2020 